\title{
EXD2 governs germ stem cell homeostasis and lifespan by promoting mitoribosome integrity and translation
}

\author{
Joana Silva1, Suvi Aivio ${ }^{1,13}$, Philip A. Knobel ${ }^{1,14}$, Laura J. Bailey², Andreu Casali', Maria Vinaixa ${ }^{3,4}$, \\ Isabel Garcia-Cao', Étienne Coyaud ${ }^{5,6}$, Alexis A. Jourdain (187,8, Pablo Pérez-Ferreros ${ }^{1,15}$, Ana M. Rojas ${ }^{10}$, \\ Albert Antolin-Fontes ${ }^{1}$, Sara Samino-Gené ${ }^{3,4}$, Brian Raught5,6, Acaimo González-Reyes ${ }^{11}$, \\ Lluís Ribas de Pouplana ${ }^{1,12}$, Aidan J. Doherty², Oscar Yanes $\mathbb{B}^{3,4}$ and Travis H. Stracker $\mathbb{1 D}^{1 \star}$
}

\begin{abstract}
Mitochondria are subcellular organelles that are critical for meeting the bioenergetic and biosynthetic needs of the cell. Mitochondrial function relies on genes and RNA species encoded both in the nucleus and mitochondria, and on their coordinated translation, import and respiratory complex assembly. Here, we characterize EXD2 (exonuclease $3^{\prime}-5^{\prime}$ domain-containing 2 ), a nuclear-encoded gene, and show that it is targeted to the mitochondria and prevents the aberrant association of messenger RNAs with the mitochondrial ribosome. Loss of EXD2 results in defective mitochondrial translation, impaired respiration, reduced ATP production, increased reactive oxygen species and widespread metabolic abnormalities. Depletion of the Drosophila melanogaster EXD2 orthologue (CG6744) causes developmental delays and premature female germline stem cell attrition, reduced fecundity and a dramatic extension of lifespan that is reversed with an antioxidant diet. Our results define a conserved role for EXD2 in mitochondrial translation that influences development and ageing.
\end{abstract}

M itochondria maintain a circular $16.6 \mathrm{~kb}$ DNA genome (mtDNA) encoding 2 ribosomal RNAs, 22 transfer RNAs and 13 open reading frames for electron transport chain (ETC) complex subunits. Mitochondria are also the major source of ATP and the site of oxidative phosphorylation (OXPHOS) and the tricarboxylic acid (TCA) cycle, which are essential for cellular respiration. In addition, mitochondria facilitate numerous metabolic reactions required for macromolecular synthesis, including the production of lipids, deoxynucleotide triphosphates, heme, iron-sulfur clusters and amino acids ${ }^{1}$

The five complexes of the OXPHOS machinery perform redox reactions using NADH and succinate to drive the majority of cellular ATP production through ATP synthase ${ }^{1}$. While most OXPHOS proteins are encoded in the nucleus, key components of all complexes, except complex II, are encoded by mtDNA. The function and maintenance of the OXPHOS machinery depends on the integrity and transcription of mtDNA, precursor RNA processing and the assembly of mitochondrial-specific ribosomes and translation ${ }^{2}$. Other factors affecting the OXPHOS system include the coordinated movement of nuclear-encoded proteins into the mitochondria, and the ordered assembly and membrane insertion of mature OXPHOS complexes that is thought to take place in close proximity to the mtDNA and mitochondrial inner membrane $^{2}$. Deficiencies in the assembly or function of the OXPHOS machinery underlies many severe human diseases ${ }^{3}$. Paradoxically, some OXPHOS defects extend lifespan in both worms and flies, in some cases in a manner that is dependent on increased reactive oxygen species (ROS) production ${ }^{4-6}$, and is classically associated with premature ageing ${ }^{1}$.

Exonuclease $3^{\prime}-5^{\prime}$ domain-containing 2 (EXD2), which is encoded in the nucleus, has previously been implicated in DNA repair and DNA damage sensitivity ${ }^{7-10}$. Here, we report that EXD2 is a mitochondrial ribonuclease that is required for mitoribosome integrity and efficient mitochondrial translation. EXD2 depletion led to widespread metabolic defects, decreased respiration and increased ROS. At the organismal level, deficiency in the Drosophila melanogaster EXD2 orthologue CG6744 resulted in developmental delays, premature female germ stem cell attrition and a dramatically extended lifespan. Collectively, our results define a critical role for EXD2 in mitochondrial homeostasis.

\footnotetext{
IInstitute for Research in Biomedicine (IRB Barcelona), The Barcelona Institute of Science and Technology (BIST), Barcelona, Spain. ${ }^{2}$ Genome Damage and Stability Centre, School of Life Sciences, University of Sussex, Brighton, UK. ${ }^{3}$ Metabolomics Platform, Department of Electronic Engineering (DEEEA), Universitat Rovira i Virgili, Tarragona, Spain. ${ }^{4}$ Biomedical Research Centre in Diabetes and Associated Metabolic Disorders (CIBERDEM), Madrid, Spain. ${ }_{5}^{5}$ Princess Margaret Cancer Centre, University Health Network, Toronto, Ontario, Canada. ${ }^{6}$ Department of Medical Biophysics, University of Toronto, Toronto, Ontario, Canada. 'Department of Molecular Biology, Howard Hughes Medical Institute, Massachusetts General Hospital, Boston, MA, USA. ${ }^{8}$ Department of Systems Biology, Harvard Medical School, Boston, MA, USA. ${ }^{9}$ Broad Institute of MIT and Harvard, Cambridge, MA, USA. ${ }^{10} \mathrm{Computational}$ Biology and Bioinformatics Group, Institute of Biomedicine of Seville (IBIS/CSIC/US/JA), Campus Hospital Universitario Virgen del Rocio, Seville, Spain. ${ }^{11}$ Centro Andaluz de Biología del Desarrollo, Universidad Pablo de Olavide/CSIC/JA, Seville, Spain. ${ }^{12}$ Catalan Institution for Research and Advanced Studies (ICREA), Barcelona, Spain. Present addresses: ${ }^{13}$ Department of Pathology, Brigham and Women's Hospital, Boston, MA, USA. ${ }^{14}$ Department for Radiation Oncology, University Hospital Zurich, Zurich, Switzerland. ${ }^{15} \mathrm{EMBL}$ Australia, University of New South Wales, Lowy Cancer Research Center, Single Molecule Science Node, Sydney and Arc Center of Excellence in Advance Molecular Imaging, Sydney, New South Wales, Australia.

*e-mail: travis.stracker@irbbarcelona.org
} 


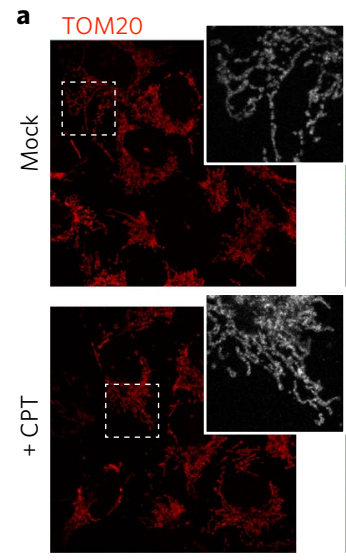

c
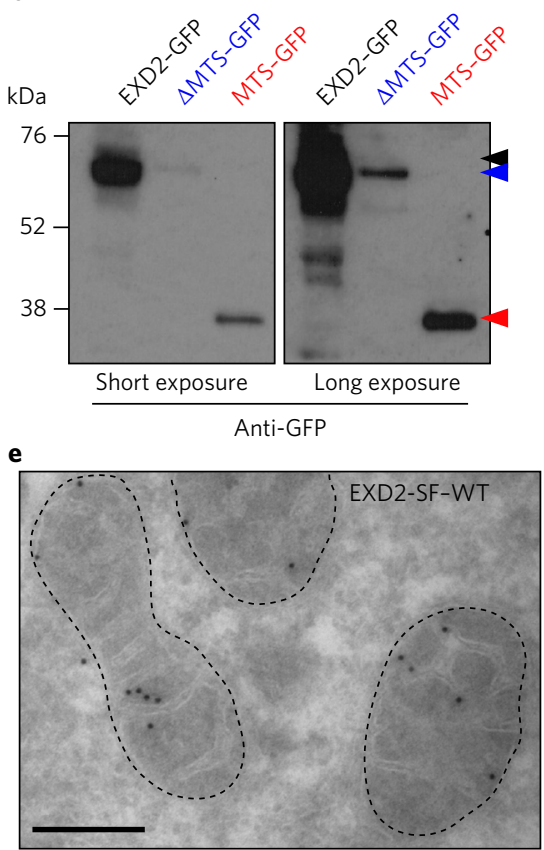
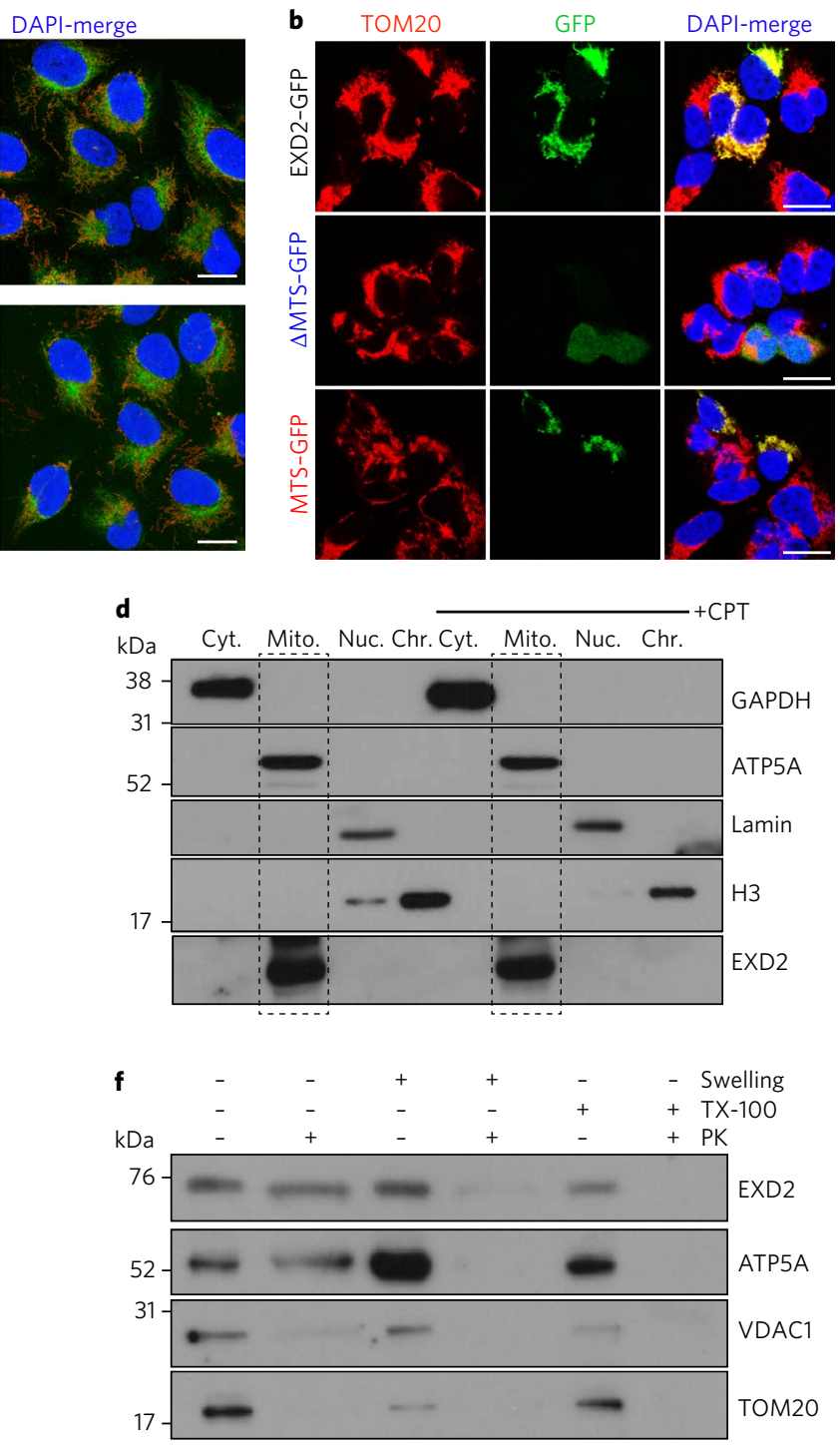

Fig. 1 | EXD2 is a mitochondrial protein. a, Immunofluorescence of endogenous EXD2 in U2OS cells revealed extensive colocalization with the mitochondrial TOM20 protein. EXD2 localization was unaltered by treatment with $1 \mu \mathrm{M}$ camptothecin (CPT) to induce DNA damage (lower panels). Scale bars, $20 \mu \mathrm{m}$. b. EXD2 contains an MTS that is necessary and sufficient for mitochondrial localization. Amino acids 1-61 of EXD2 are sufficient to localize GFP to mitochondria (bottom panels). Deletion of amino acids 1-61 of EXD2 leads to diffuse nuclear-cytoplasmic localization (middle panels). Scale bars, $15 \mu \mathrm{m}$. Images in $\mathbf{a}$ and $\mathbf{b}$ represent 1 out of 5 experiments. $\mathbf{c}$, Western blot of GFP fusion proteins with expected sizes indicated. Note poor expression and destabilization of $\triangle$ MTS-GFP. Arrowheads indicate the protein of the corresponding label colour. $\mathbf{d}$, Subcellular fractionation (cytoplasm (Cyt.), mitochondria (Mito.), nucleus (Nuc.) and chromatin (Chr.)) of EXD2 (enclosed by dashed boxes) in the presence or absence of $1 \mu \mathrm{M}$ CPT indicated exclusively mitochondrial localization. Data represent 1 out of 3 experiments. e, TEM indicated mitochondrial (dashed outlines) localization of EXD2-SFWT. Scale bar, $200 \mathrm{~nm}$. See Supplementary Fig. 1f,g for additional examples. f, Western blot of mitochondria isolated from U2OS cells treated with $50 \mu \mathrm{g} \mathrm{ml}^{-1} \mathrm{PK}$ in the presence or absence of Triton X-100 shows that EXD2 is resistant, similar to the OXPHOS subunit ATP5A, indicating inner membrane and/or matrix localization. Data in e,f represent 1 out of 2 experiments. Uncropped blots are shown in Supplementary Fig. 9.

\section{EXD2 is a mitochondrial protein}

EXD2 encodes a $3^{\prime}-5^{\prime}$ exonuclease (EXO) domain similar to one encoded by $W R N$, a gene in which mutations cause Werner's syndrome progeria ${ }^{11}$ (Supplementary Fig. 1a). Additionally, EXD2 has a highly conserved carboxy-terminal $\mathrm{HNH}$ endonuclease-like domain characterized by tandem CXXC motifs (Supplementary Fig. 1b-d). As previous reports have suggested a role for EXD2 in DNA repair ${ }^{7-9}$, we examined the localization of this exonuclease. EXD2 was predominantly located in the cytoplasm, regardless of camptothecin-induced DNA damage, and overlapped substantially with mitochondrial TOM20 (Fig. 1a). Comparative sequence analysis identified a putative, amino-terminal mitochondrial targeting sequence (MTS) conserved in most EXD2 homologues identified so far (Supplementary Fig. 1e). Overexpression of green fluorescent protein (GFP)-tagged EXD2 (EXD2-GFP) or of its MTS fused to GFP (MTS-GFP; amino acids 1-61) resulted in a mitochondrial GFP signal, whereas the $\triangle$ MTS-GFP (in which amino acids 1-61 are deleted) protein was diffusely nucleocytoplasmic and destabilized (Fig. 1b,c). Subcellular fractionation for endogenous EXD2 (Fig. 1d) and transmission electron microscopy (TEM) of cells stably expressing streptavidin and FLAG (SF)-tagged wild-type EXD2 (EXD2-SF-WT) confirmed that EXD2 primarily localized to mitochondria (Fig. 1e and Supplementary Fig. 1f,g). In contrast to the outer membrane proteins TOM20 or VDAC1, which are susceptible to proteinase-K (PK)-mediated digestion, EXD2 was resistant, a response that is 
similar to that of the inner membrane ATP5A subunit of ATP synthase (Fig. 1f). In addition, while treatment with carbonyl cyanide-4-(trifluoromethoxy)phenylhydrazone (FCCP) liberated TOM20 from the outer membrane, EXD2 remained relatively resistant. This result is similar to ETC components that localize to the inner membrane or matrix (Supplementary Fig. 1h). Together, these results indicate that EXD2 is an intra-mitochondrial protein targeted by an N-terminal MTS.

\section{EXD2 influences cellular metabolism}

As mitochondria play a key role in metabolism, we examined the impact of EXD2 deficiency using complementary metabolomics approaches ${ }^{12,13}$. EXD2 depletion (Fig. 2a,b) led to alterations in many metabolites, including those of the TCA cycle, antioxidants and glycolysis (Fig. 2c). Using the NMR-based positional enrichment by proton analysis (PEPA) technique, we followed ${ }^{13} \mathrm{C}$ isotope incorporation of glucose or glutamine, which are key precursors for numerous metabolites of central carbon metabolism ${ }^{13,14}$. Pathway enrichment analysis revealed significant alterations in several metabolite classes, including the glutathione cycle, amino acid and sugar metabolism, the pentose phosphate pathway and the TCA cycle (Fig. 2d). Moreover, we observed marked defects in the incorporation of glucose-derived carbon into many metabolites, whereas glutamine was relatively unaffected (Fig. 2e,f). These data indicate that EXD2 is required for glucose oxidation through the TCA cycle and glutathione synthesis, suggesting that EXD2-deficient cells may have alterations in ROS or antioxidant capacity and rely on glutamine for the generation of key metabolites.

\section{EXD2 prevents ROS and is required for OXPHOS activity}

To understand the abovementioned effects, we generated EXD2knockout (KO) cell lines using CRISPR-Cas9 technology and complemented them with EXD2-SF-WT, an EXO domain mutant (NUC) or HNH-like domain mutants $(\mathrm{C} 1 \text { and } \mathrm{C} 2)^{15}$ (Supplementary Figs. 1a,c and 2a). EXD2-deficient cells had reduced glutathione levels (Fig. 3a and Supplementary Fig. 2b), consistent with metabolomics data (Fig. 2), and increased levels of ROS (Fig. 3b). Expression of EXD2-SF-WT or treatment with the antioxidant $\mathrm{N}$-acetylcysteine (NAC), but not with the EXD2-SF-NUC and C2 mutants, rescued ROS levels to that of wild-type cells (Fig. 3b). EXD2-deficient cells also exhibited reduced levels of mtDNA (Fig. $3 c$ and Supplementary Fig. 2c,d), a phenotype associated with increased ROS levels and defective respiration ${ }^{16}$. mtDNA reduction was independent of clear changes in mitochondrial mass (Fig. 3d,e and Supplementary Fig. 2e) or mtDNA replication (Supplementary Fig. 2f) and could be rescued with expression of EXD2-SF-WT and partially with NAC treatment (Fig. $3 \mathrm{c}$ and Supplementary Fig. 2d).

Growth of EXD2-deficient cell lines was indistinguishable from wild-type parental U2OS cells cultured in rich medium containing glucose and glutamine (Fig. 3f). However, we observed impaired growth of EXD2-KO1 cells cultured in media lacking glutamine (Fig. $3 g$ and Supplementary Fig. 2g), which was restored by treatment with EXD2-SF-WT but not EXD2-SF-NUC or C2 mutants (Fig. 3g). Moreover, NAC did not rescue the impaired growth, indicating that the growth defects of EXD2-KO1 cells were not secondary to increased ROS production or sensitivity to hyperoxic culture conditions (Fig. 3h,i).

Mitochondrial ROS originates predominantly from complexes I and III of the ETC ${ }^{17}$, so we examined ETC function by measuring oxygen consumption rates (OCRs) in both U2OS and MDA-MB-231 cells. Compared with parental or EXD2-SF-WT complemented cells, EXD2-KO1 cells exhibited reduced OCRs and ATP-linked respiration that could not be efficiently rescued by EXD2 mutants (Fig. $3 \mathrm{j}$ and Supplementary Fig. $2 \mathrm{~h}$ ). In addition, extracellular acidification rates (ECARs) were elevated in
EXD2-KO or mutant cells, indicative of increased aerobic glycolysis and lactate production that can accompany defective OXPHOS ${ }^{18}$ (Fig. 3k and Supplementary Fig. 2i). NAC partially rescued overall OCRs, but mitochondrial ATP-linked respiration remained impaired, suggesting that reduced OXPHOS capacity could be one of the primary defects leading to increased ROS levels (Fig. 31 and Supplementary Fig. 2j).

Metabolomics data indicated that the reduced OCRs could reflect impaired glucose usage in EXD2-deficient cells (Fig. 2e,f). We tested this hypothesis using specific media and observed an increase in OCRs following glucose addition in cells lacking functional EXD2 (Fig. 3m). To more directly confirm a role for EXD2 in ATP production, we analysed ATP levels using multiple methods and observed that EXD2-KO1 cells had reduced levels of ATP that were rescued by EXD2-SF-WT expression (Fig. 3n and Supplementary Fig. 2k,l). Moreover, these defects were not rescued by NAC (Fig. 3n and Supplementary Fig. 2k). We therefore concluded that EXD2 deficiency results in impaired mitochondrial ATP production, ROS accumulation, reduced mtDNA levels and glutamine-dependent cell growth.

\section{EXD2 associates with complex I and the mitoribosome}

To determine how EXD2 influences metabolism, we used BioID mass spectrometry (BioID-MS) to identify proximity interactors ${ }^{19}$. EXD2-WT and EXD2-C2 fused to a FLAG-tagged BirA* biotin ligase (EXD2-BirA*-WT and EXD2-BirA*-C2, respectively) were overexpressed in the presence of biotin in human AD293 cells. While BirA* expression resulted in staining throughout the cell, the streptavidin signal was only detected in the cytoplasm of biotin-supplemented cells expressing EXD2-BirA*-WT or EXD2-BirA ${ }^{*}-\mathrm{C} 2$, and this expression pattern colocalized extensively with TOM20 (Fig. 4a). BioID-MS revealed an EXD2 proximity interactome composed almost entirely of mitochondrial proteins (Fig. $4 \mathrm{~b}$ and Supplementary Table 2 ) and highly enriched for components of complex I and the small $28 \mathrm{~S}$ subunit of the mitoribosome (Fig. 4c). The components included almost all of the matrix-exposed complex I subunits ${ }^{20}$, more than 10 subunits of the $28 \mathrm{~S}$ mitoribosome, proteins involved in RNA processing or translation and assembly (for example, GRSF1, TACO1, ACAD9 and LETM1), as well as enzymes implicated in OXPHOS substrate generation (for example, MDH2 and GLS) (Fig. 4c). Most interactions were also observed with EXD2-C2, which fails to complement phenotypes of EXD2-KO1 cells. However, the representation of $28 \mathrm{~S}$ components was specifically reduced compared with EXD2-WT (Fig. 4c and Supplementary Table 2), suggesting that the HNH-like domain influences the frequency or affinity of this subset of interactors.

An interaction with the complex I subunit NDUFA9 was observed in immunoprecipitation (IP)-western blot analyses, EXD2-KO1 cells showed minor reductions in the levels of complex I, supercomplexes of complexes I and III, and, to a lesser extent, complex V (ATP synthase) and complex I-dependent OCR was also reduced (Supplementary Fig. 3a-d). As EXD2 did not appear to be present in fully assembled complex I (Supplementary Fig. 3b), we analysed the mitoribosome components and readily observed an interaction with DAP3 (28S subunit) that was impaired by the EXD2-SF-C2 mutant (Fig. 4d). In contrast, we did not see an interaction with the large mitoribosome (39S) subunit MRPL3 or with VDAC1, an outer mitochondrial membrane protein, in IP-western blots (Fig. 4d). The mitoribosome association was further validated by confocal microscopy, whereby extensive colocalization of endogenous EXD2 and MRPL12 was observed (Fig. 4e). This result further established the predominant mitochondrial localization of EXD2 and suggested that its primary functions could be linked to the OXPHOS machinery and to the mitoribosome. 

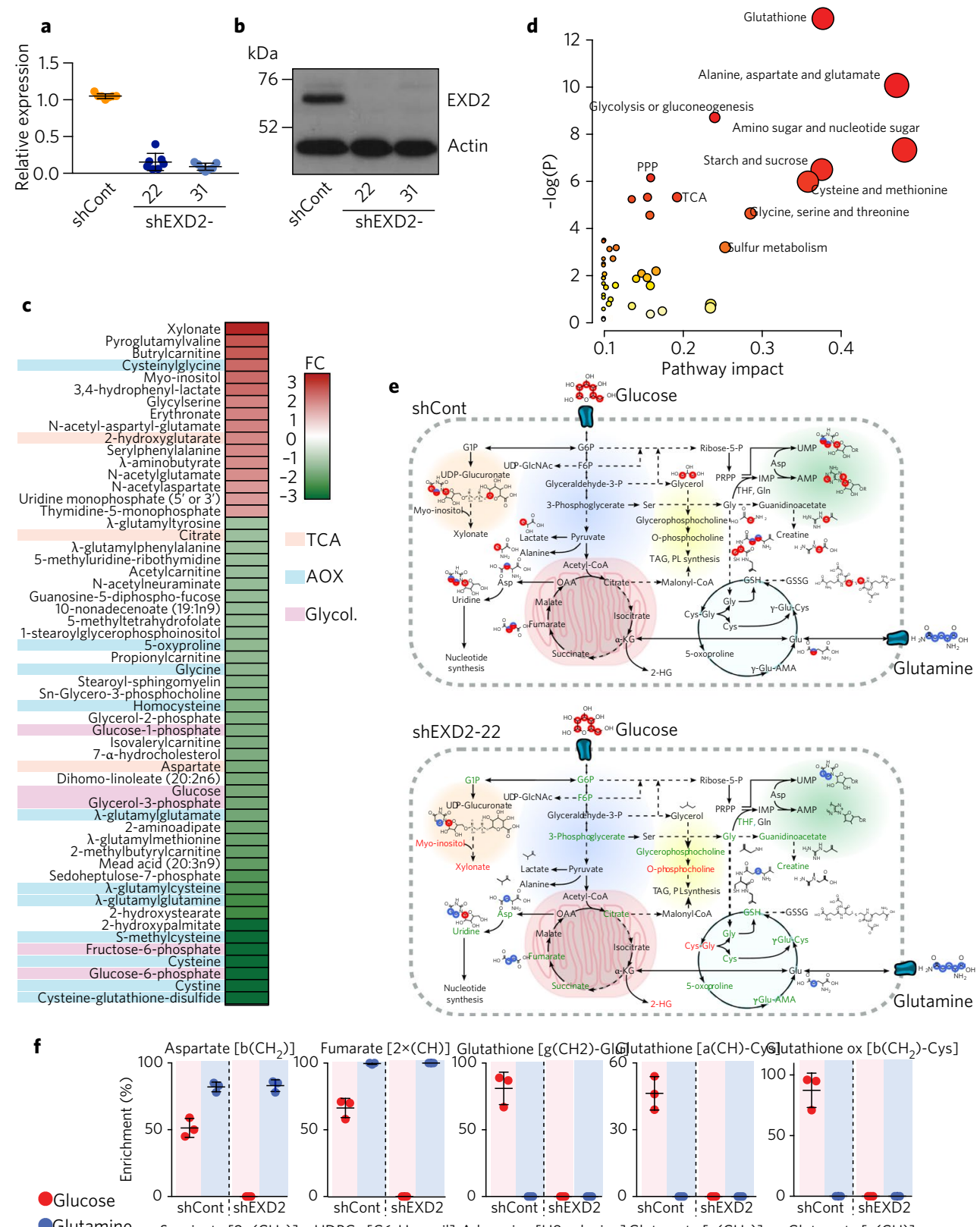

Glutamine Succinate $\left[2 \times\left(\mathrm{CH}_{2}\right)\right]$ UDPGs [C6-H uracil] Adenosine [H8-adenine] Glutamate $\left[\mathrm{g}\left(\mathrm{CH}_{2}\right)\right] \quad \mathrm{Glutamate}[\mathrm{a}(\mathrm{CH})]$
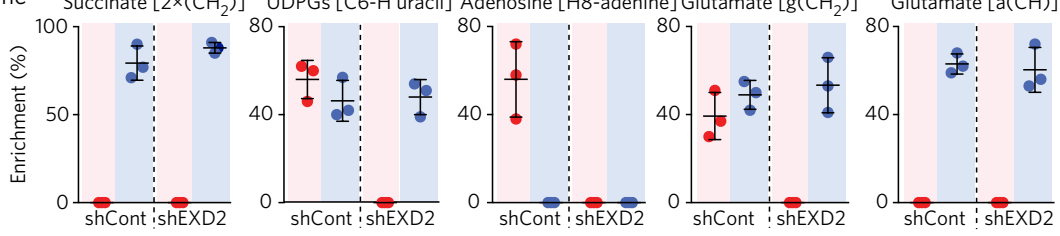

Fig. 2 | EXD2 is required for metabolic homeostasis. a, qRT-PCR confirmed the reduction of EXD2 mRNA following expression of stable shRNAs against EXD2. Averages from 7 independent experiments in triplicate are plotted $(n=7)$. b. Confirmation of reduced EXD2 protein levels in cells expressing shRNAs against EXD2. Actin serves as a loading control. Data represent 1 out of 5 experiments. c, Heatmap representation of the fold change (FC) in metabolites that are significantly altered by EXD2 (shEXD2-22) depletion compared with control (shCont) cells. Values represent the means of $n=5$ independent samples. Depicted metabolites have a FC $>1.3$ with an adjusted $P<0.01$ ( $t$-test). Metabolites involved in the tricarboxylic acid (TCA) cycle, antioxidant generation (AOX) and glycolysis (Glycol.) are indicated. $\mathbf{d}$, Pathway enrichment analysis of metabolite differences from MS data and ${ }^{1} \mathrm{H}-\mathrm{NMR}$ metabolite profiling. The $x$ axis represents pathway impact calculated from topological analysis based on the centrality measures of individual metabolites in a given metabolic network and the $y$ axis represents false discovery rate (FDR) $P$ values from a hypergeometric test. Overrepresented pathways with higher impact are highlighted with darker colours and larger sizes. e, Metabolic summary chart showing active pathways in U2OS cell lines, as revealed by ${ }^{1} \mathrm{H}$-NMR PEPA ${ }^{13}$. Metabolic structures are depicted with red and blue dots indicating fates of $\left[\mathrm{U}_{-}{ }^{13} \mathrm{C}\right]$ glucose and $\left[\mathrm{U}-{ }^{13} \mathrm{C}\right]$ glutamine, respectively. The $\mathrm{FCs}$ of metabolites identified from both approaches are indicated in red (up) or green (down) text in accordance with the heatmap. $\mathbf{f}$, EXD2 depletion impairs glucose usage. Examples of ${ }^{1} \mathrm{H}-\mathrm{NMR}$ metabolite profiling predicted significant fractional enrichments. Red and blue dots represent individual $F$ values calculated using $\left[\mathrm{U}-{ }^{13} \mathrm{C}\right]$ glucose and $\left[\mathrm{U}-{ }^{13} \mathrm{C}\right]$ glutamine, respectively. Results from $n=3$ independent cultures plotted as the mean (s.d.) with individual values shown. Source data are provided in Supplementary Table 1 and unprocessed scans for $\mathbf{b}$ are provided in Supplementary Fig. 9. 
a
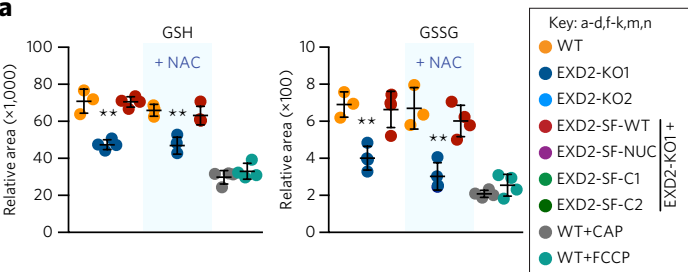

c

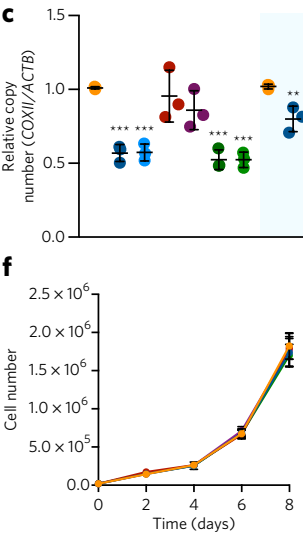

d

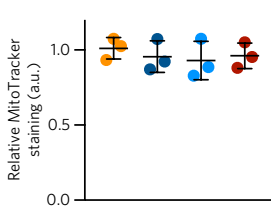

b

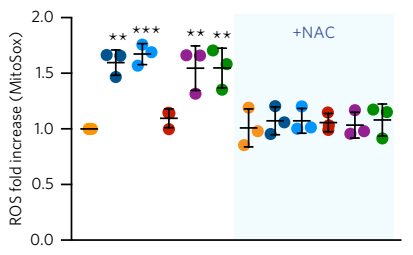

e
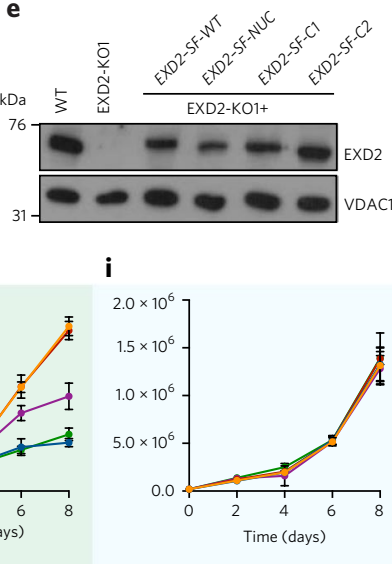

j
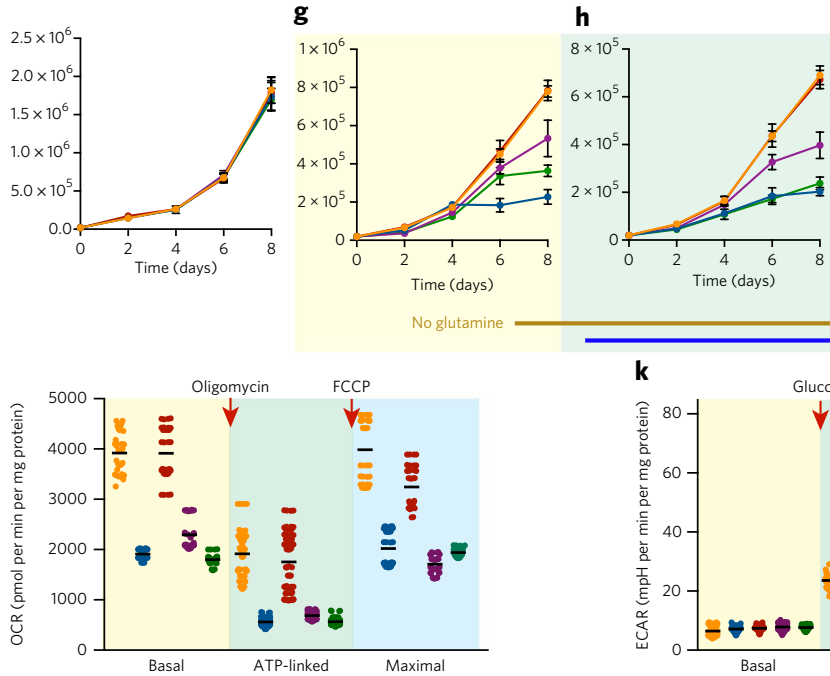

i

k

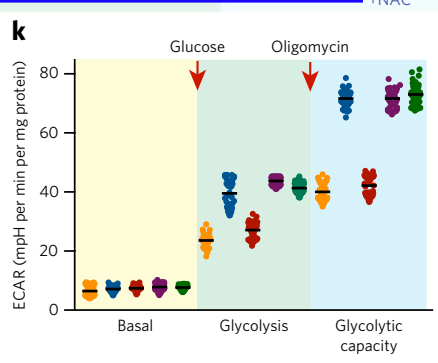

I
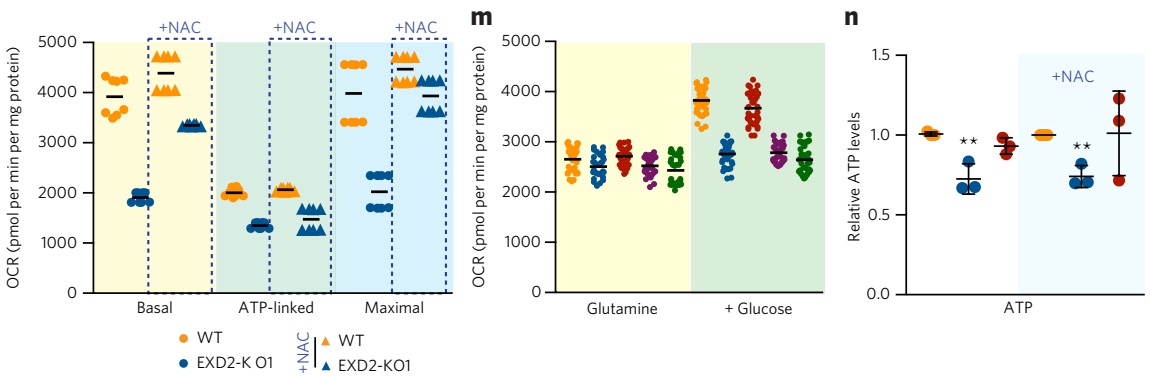

Fig. 3 | Characterization of EXD2-deficient cells. a, LC-MS analysis of glutathione (GSH or GSSG) levels in MDA-MB-231 cells in the presence or absence of NAC or with CAP or FCCP. Values of $n=3$ (wild type) or $n=4$ (all others) independent cultures with means (s.d.) (**P<0.01, unpaired $t$-test, two-tailed). The colour legend applies to all panels in the figure. $\mathbf{b}$, ROS levels in MDA-MB-231 cells measured with MitoSOX red. Controls and gating examples are provided in Supplementary Fig. 8. Averages of $n=3$ independent experiments with means (s.d.) ${ }^{\star \star} P<0.01,{ }^{\star \star \star} P<0.001$, unpaired $t$-test, two-tailed). c, mtDNA levels determined using qRT-PCR in U2OS cells in the presence or absence of NAC. Averages of $n=3$ independent experiments with means (s.d.). Similar results obtained in multiple cell lines (Supplementary Fig. 2c,d). Statistical analysis of b,c: one-way ANOVA with Dunnet's test ( ${ }^{\star \star \star} P<0.001$, $\left.{ }^{\star \star} P<0.01,{ }^{\star} P<0.05\right)$. d, Mitochondrial mass measured by MitoTracker Green FM staining of U2OS cells. Averages of $n=3$ independent experiments with the means (s.d.) plotted. e, Mitochondrial mass measured by western blotting for VDAC1 levels. Data represent 1 out of 3 experiments. $\mathbf{f}-\mathbf{i}$, EXD2-deficient cells grow normally in rich media (f) but glutamine removal severely impaired growth ( $\mathbf{g}$ ). NAC did not rescue growth in the absence of glutamine (h) but slightly reduced growth in all lines (i). The means (s.d.) of $n=3$ independent experiments are shown. $\mathbf{j}, \mathbf{k}$, OCR analysis (mitochondrial stress test: oligomycin, FCCP and antimycin plus rotenone) (j) revealed decreased basal, ATP-linked and maximal respiration in EXD2-deficient cells and increased ECAR (glycolysis stress test: glucose, oligomycin and 2-deoxy-D-glucose) (k) that was rescued by EXD2-SF-WT but not NUC or C2 mutants in U2OS cells. Red arrows indicate treatment between category measurements. All time course values from quadruplicate cultures of two independent experiments with the means are shown for j and $\mathbf{k}$. I, Influence of NAC on OCR. Values from quadruplicate cultures of two independent experiments with the means are shown. Similar results in MDA-MB-231 are shown in Supplementary Fig. 3h-j. $\mathbf{m}$, U2OS cells treated with glucose to distinguish OCR from glutaminolysis or glycolysis. All time course values from quadruplicate cultures of $n=2$ independent experiments with the means are shown. $\mathbf{n}$, EXD2-KO1 U2OS cells exhibited lower ATP levels than wild-type or complemented cells. Averages from $n=3$ independent cultures are shown with the means (s.d.) ${ }^{\star \star} P<0.0070$ and 0.0029 , unpaired $t$-test, two-tailed). Source data and statistical information are provided in Supplementary Table 1, and uncropped western blots are provided in Supplementary Fig. 9. 
a
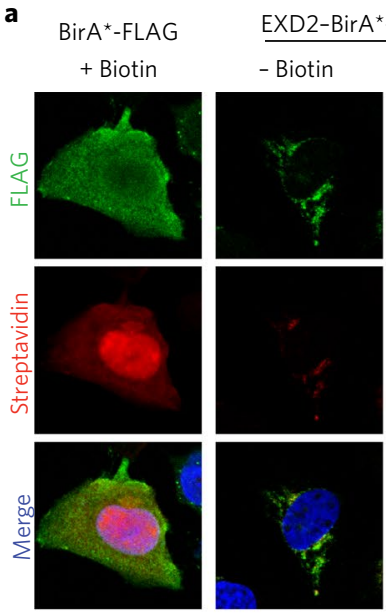
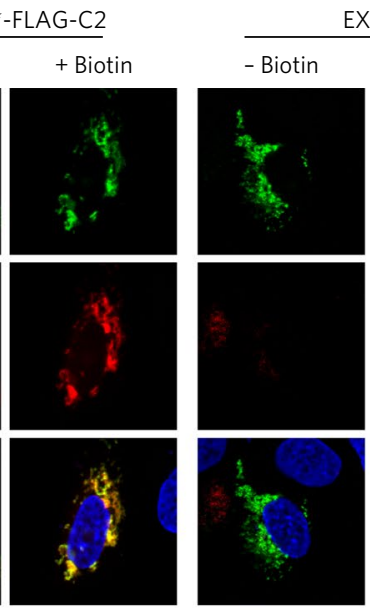

EXD2-BirA*-FLAG-WT
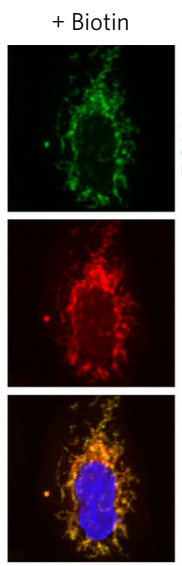
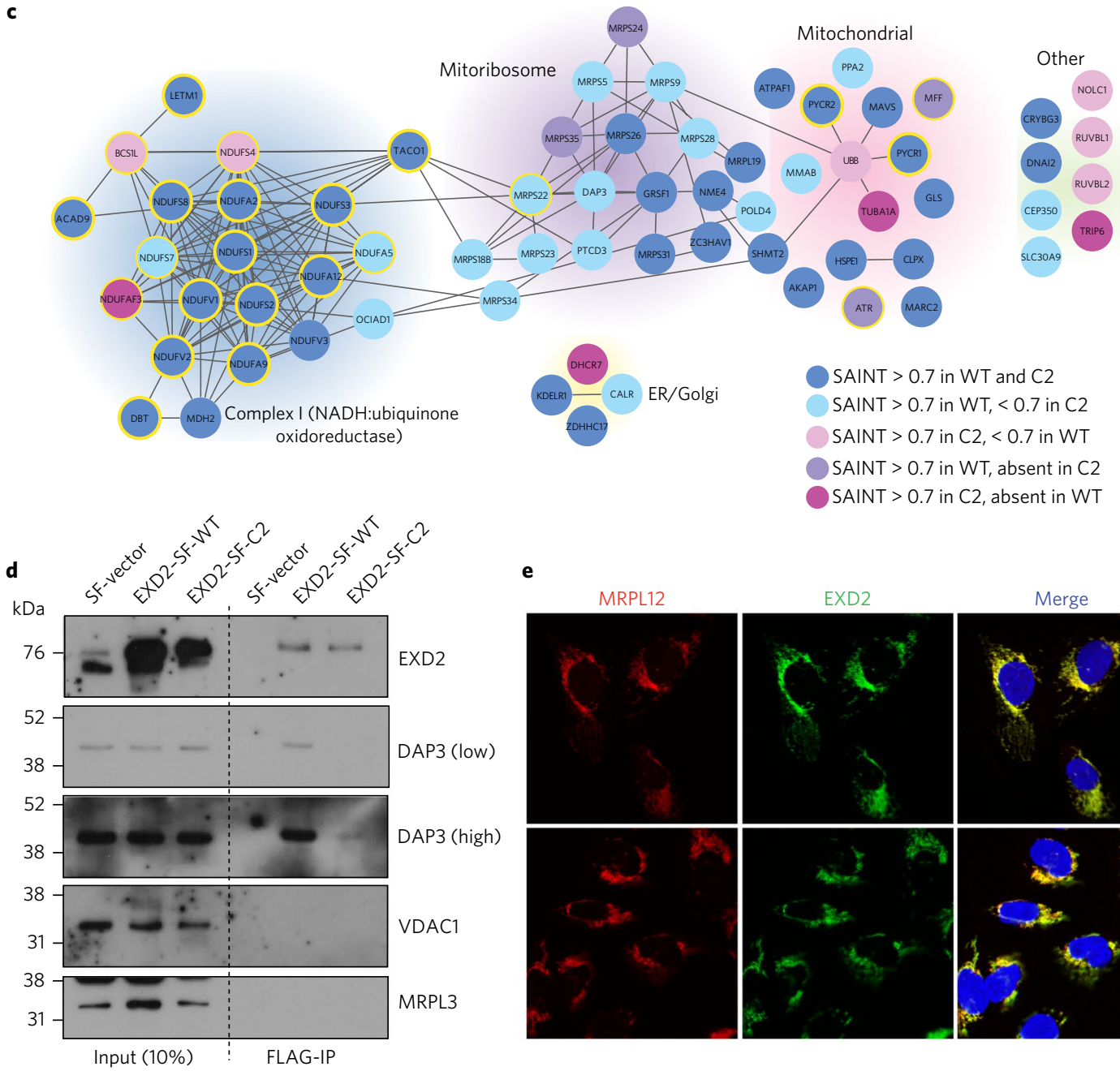
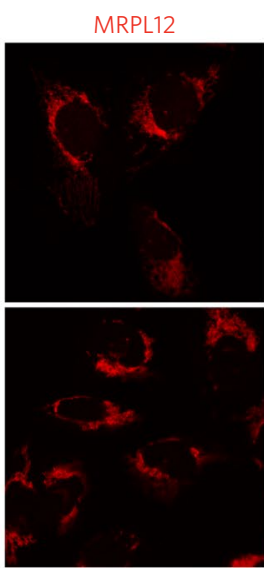

EXD2
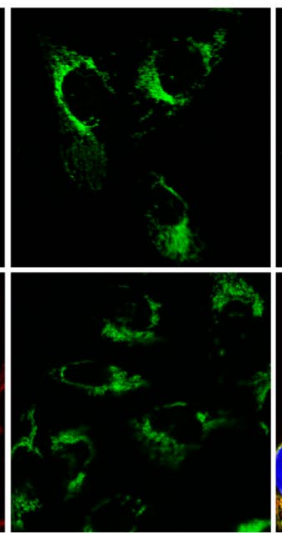

b

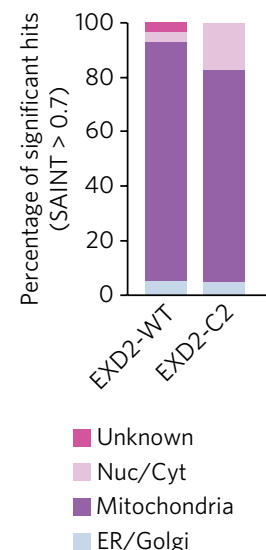

Other

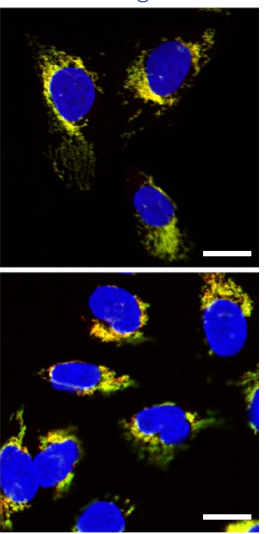

Fig. 4 | EXD2 interacts with complex I and the mitoribosome. a, Expression of FLAG-tagged BirA*, EXD2-BirA*-C2 or EXD2-BirA*-WT fusion proteins with or without $24 \mathrm{~h}$ of biotin labelling. Bir $A^{\star}$-expressing control cells exhibited a diffuse nucleo-cytoplasmic streptavidin signal, whereas streptavidin staining was predominantly cytoplasmic for EXD2-Bir $A^{\star}-C 2$ or EXD2-BirA*-WT and overlapped extensively with the mitochondrial marker TOM20. Scale bar, $10 \mu \mathrm{m}$. b, Graphical representation of the subcellular localization of proximity interactors in EXD2-BirA*-WT- or EXD2Bir $A^{\star} C 2$-expressing cells. Summarized results are provided in Supplementary Table 2. c, EXD2 primarily associates with mitochondrial proteins, and interactors are enriched for complex I and the mitoribosome. Proteins are colour-coded based on the significance analysis of interactome (SAINT) score (see legend and Supplementary Table 2), and those involved in human hereditary diseases are circled in yellow. d, EXD2 interacts with DAP3 based on IP-western blots, but not with VDAC1 or MRPL3, and the interaction is severely reduced in cells expressing the EXD2-C2 mutant. Results from IP-western blotting of U2OS cell extracts are shown. Data represent 1 out of 3 experiments. Uncropped western blots are provided in Supplementary Fig. 9. e, Immunofluorescence analysis of endogenous human EXD2 in U2OS cells by confocal microscopy revealed extensive colocalization with the mitoribosome protein MRPL12. Scale bars, $10 \mu \mathrm{m}$. Images in a,e represent more than 25 cells analysed in two experiments. 


\section{EXD2 facilitates mitochondrial translation}

We next examined mitoribosome integrity using sucrose gradient fractionation. EXD2 co-fractionated with the $28 \mathrm{~S}$ subunit and with the fully assembled $55 \mathrm{~S}$ mitoribosome, but not with the free 39 S subunit (Fig. 5a, top panels). As early 28S association of EXD2 suggested that EXD2 has a potential role in or during assembly, we analysed ribosome integrity in EXD2-KO1 cells and in cells expressing EXD2-SF-C2. In both cases, we observed a clear shift of the 28S DAP3 subunit into higher molecular mass complexes overlapping with $39 \mathrm{~S}$ fractions (Fig. $5 \mathrm{a}$, middle and bottom panels). RNA analyses revealed increased $12 \mathrm{~S}$ rRNA and mtDNA encoded mRNAs in the aberrant fractions, suggesting that a premature mitochondrial mRNA association with the $28 \mathrm{~S}$ could yield the larger, aberrant complexes (Fig. 5b, compare top panels with middle and bottom panels). Consistent with this possibility, the profiles of RNase A-treated fractions were similar regardless of EXD2 status (Supplementary Fig. 4a). The EXD2-SF-C2 mutant protein did not visibly co-fractionate with the free $28 \mathrm{~S}$ subunits, a result that is consistent with the $\mathrm{HNH}$-like domain promoting the $28 \mathrm{~S}$ association (Fig. 5a, bottom panel).

The appearance of aberrant $28 \mathrm{~S}$ subunits suggested that mitochondrial translation could be impaired; therefore we analysed this possibility using metabolic pulse labelling ${ }^{21}$. A strong reduction in global mitochondrial translation rates was observed in U2OS and RPE1 cells lacking EXD2 (Fig. $5 \mathrm{c}-\mathrm{f}$ and Supplementary Fig. 4b-e); this effect was complemented by EXD2-SF-WT, but not by EXD2SF-NUC or C2 mutants. Furthermore, NAC treatment did not influence the phenotype (Fig. 5f), suggesting that this effect was likely a direct result of EXD2 loss. Together, these data indicate that EXD2 prevents inappropriate mRNA association with mitoribosomes and facilitates efficient mitochondrial translation that requires both its EXO and HNH-like domains.

\section{EXD2 preferentially binds single-stranded RNA}

As EXD2 loss appeared to influence the association of mRNA with mitoribosomes, we considered that EXD2 may function as an RNase. We examined the activity and specificity of bacterially purified EXD2 and that of the WRN exonuclease domain (WRNEXO) for comparison. The MTS of EXD2 (1-61) was removed to improve solubility and we generated the EXD2-NUC mutant (to control for contaminating nucleases) and the EXD2-C2 mutant (as it fails to complement EXD2-KO1 cells) (Fig. 6a and Supplementary Fig. 5a). As previously reported ${ }^{8}$, EXD2-WT, but not EXD2-NUC exhibited $3^{\prime}-5^{\prime}$ exonuclease activity on single-stranded (ssDNA) (Supplementary Fig. 5b). In addition, both EXD2-WT and WRNEXO exhibited activity on double-stranded DNA (dsDNA), ssRNA, both strands of a DNA-RNA hybrid and several other DNA substrates (Fig. 6b-d and Supplementary Fig. 5d-f). Similar activity was observed with EXD2-C2, although it was consistently lower, indicating that stability or selectivity of the enzyme-nucleic acid complex may be affected (Fig. 6e and Supplementary Fig. 5f-g). However, when $\mathrm{MgCl}_{2}$ was used as a cofactor, both EXD2-WT and EXD2-C2, but not the closely related WRN-EXO, showed stronger substrate discrimination, with exonuclease cleavage observed only on ssRNA or RNA-DNA hybrids (Fig. 6f-i and Supplementary Fig. 5h-k). Quantification of multiple experiments revealed that the specific activity of EXD2 increased by approximately threefold on ssRNA compared with ssDNA when using $\mathrm{MnCl}_{2}$. In contrast, in the presence of $\mathrm{MgCl}_{2}$, EXD2 activity on ssDNA and dsDNA substrates was lost, reduced on hybrids, but similar on ssRNA (Fig. 6j and Supplementary Fig. 5l).

To determine whether EXD2 had any binding preference, we performed electrophoretic mobility shift assays (EMSAs) on fluorescently labelled substrates. We observed a clear supershift with ssRNA, but not with ssDNA or hybrid molecules, when EXD2 was added (Fig. 6k). Similarly, EXD2-NUC and EXD2-C2 mutants bound preferentially to ssRNA, although the supershift was reduced with EXD2-C2 (Fig. 61,m), and WRN-EXO did not exhibit any obvious substrate preference (Supplementary Fig. $5 \mathrm{~m}$ ). Together, these data demonstrate that EXD2 and WRN have promiscuous $\mathrm{MnCl}_{2}-$ dependent activity on various RNA and DNA substrates in vitro. However, EXD2 preferentially binds to ssRNA and can maintain its activity on ssRNA in the presence of $\mathrm{MgCl}_{2}$, indicating that it is more likely to be the physiological substrate of its activity.

\section{CG6744 influences Drosophila metabolism and development} Given the influence of EXD2 on mitochondrial function, we examined the impact of depleting the D. melanogaster EXD2 orthologue CG6744 (henceforth referred to as dEXD2) (Supplementary Fig. 6a). Consistent with available data ${ }^{22}$, two peaks of $d E x d 2$ expression were observed in control yellow white flies in early embryos (0-4 h) and in pupae (WPP) (Supplementary Fig. 6b,c), which are developmental phases associated with high metabolic activity $^{23}$. We obtained flies with a transposon insertion and confirmed $d E x d 2$ mRNA depletion in homozygous animals (Fig. 7a). Similar to human cells, mtDNA levels were lower in mutant animals and largely rescued by NAC treatment (Fig. 7b), and mitochondrial translation rates were reduced in dEXD2-deficient flies (Fig. 7c,d). Moreover, overexpressed dEXD2 localized extensively to the mitochondria of Drosophila Schneider 2 (S2) cells (Fig. 7e), and dEXD2-deficient flies exhibited impaired respiration (Fig. 7f) and lower levels of ATP and glutathione (Fig. 7g). Embryos and pupae lacking dEXD2 were smaller than controls but appeared morphologically normal, suggesting a developmental delay (Fig. 7h). In addition, pupariation times were extended, but could be normalized by NAC treatment or further extended with paraquat treatment, which elevates ROS levels (Fig. 7i). These effects were not due exclusively to maternal inheritance of defective mitochondria, as the expected ratio of normal to runt animals was observed following breedings from heterozygous females, which transmit intact mitochondria to all offspring, with homozygous males (Supplementary Fig. 6d).

\section{EXD2 prevents germ stem cell attrition and regulates lifespan} As mitochondrial dysfunction and ROS extend or diminish lifespan in different experimental settings ${ }^{4-6}$, we examined the lifespan of dEXD2-deficient animals. We observed a slight but significant lifespan extension in males that was more dramatic in females and normalized by NAC treatment (Fig. 8a and Supplementary Table 1). In adult flies, expression of $d E x d 2$ was higher in the ovary than in the brain or gut (Fig. 8b), and mutants exhibited an accelerated reduction in fecundity that was largely rescued by NAC treatment (Fig. 8c). To ascertain the origin of this phenotype, we examined the pool of germ stem cells (GSCs) present in control and dEXD2-deficient ovaries. As previously described, a progressive GSC decline in control flies was observed ${ }^{24}$, a phenotype that was accelerated in dEXD2-deficient animals and rescued by NAC treatment (Fig. 8d,e). Cytological analyses of dEXD2-deficient ovaries revealed a disorganized mitochondrial network with highly reduced levels of cytochrome $c$, an essential component of the ETC, suggesting a metabolic collapse in the germarium where the GSCs and somatic follicle stem cells reside (Fig. 8f). These results suggest that EXD2 is crucial for mitochondrial homeostasis in vivo and that it prevents excess ROS production that retards normal development and causes the premature attrition of GSCs, reduced fecundity and extended lifespan ${ }^{25}$ (Supplementary Fig. 6e).

\section{Discussion}

Our data connects the EXO and $\mathrm{HNH}$-like domains of EXD2 to mitochondrial ribosome integrity, translation, OXPHOS activity and Drosophila germline stem cell maintenance and lifespan. 
a

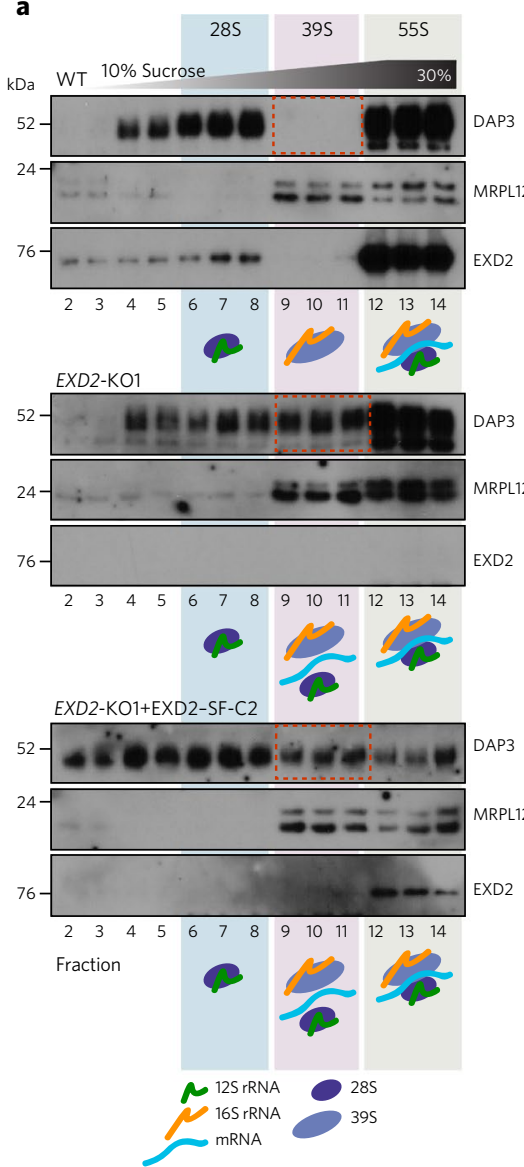

c

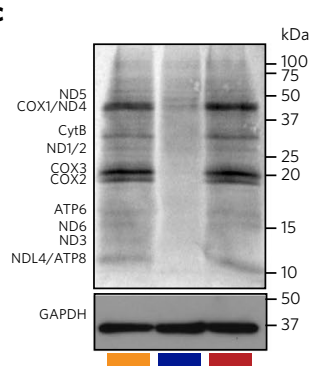

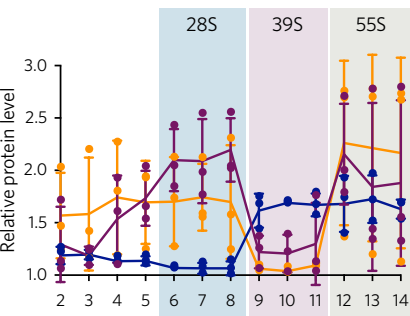

b
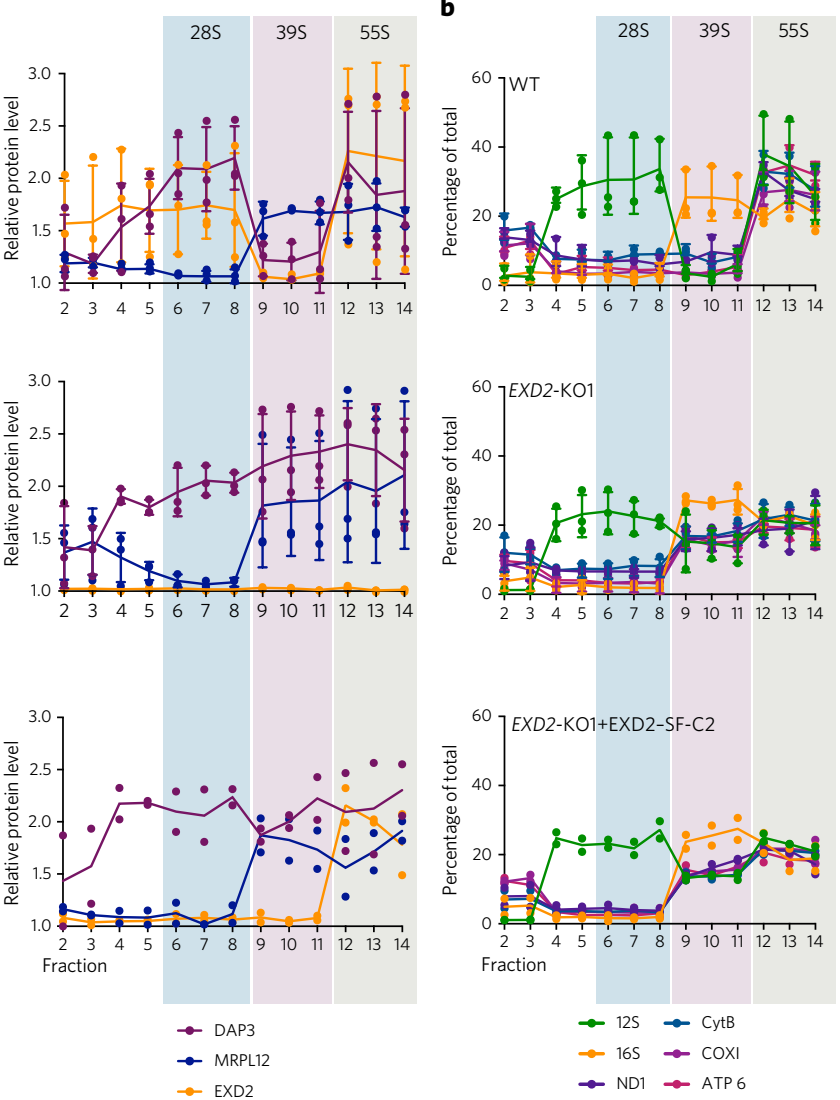

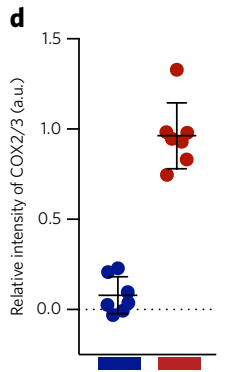

e
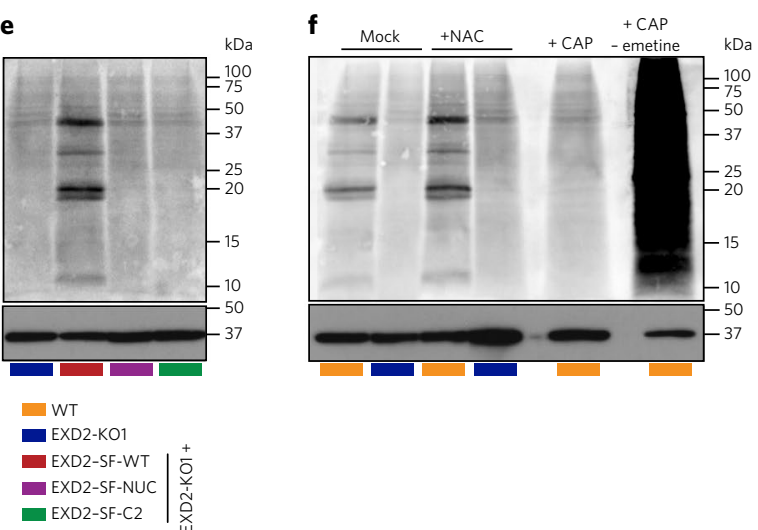

Fig. 5 | EXD2 prevents aberrant mRNA association with the mitoribosome and facilitates translation. a, Sucrose gradient fractionation shows the distribution of markers of the 28S (DAP3) and 39S (MRPL12) mitoribosome. EXD2 co-sediments with the 28S and assembled 55S mitoribosome. EXD2-KO1 and EXD2-SF-C2 cells show differences in 28S distribution (compare red boxes). Quantification shown for wild-type and EXD2-KO1 cells (right) with individual values of three independent experiments shown with the means (s.d.). Quantification of EXD2-SF-C2 with individual values of $n=2$ independent experiments shown with the means. Schematic representation of detected components is shown below each plot. $\mathbf{b}$, qRT-PCR analysis of the sedimentation profile of the indicated mitochondrial transcripts in wild-type, EXD2-KO1 and EXD2-SF-C2 cells. The abundance of transcripts in each fraction is shown as a percentage of the total levels. The distribution of fractions containing the 28S, 395 and 55S detected by western blotting is shown for reference. Quantification of $n=3$ independent experiments is shown for wild-type and EXD2-KO1 cells with all values and the mean (s.d.) indicated. Quantification of $n=2$ independent experiments is shown for EXD2-C2 with individual values and the mean indicated. c, EXD2 is required for efficient mitochondrial translation. Phosphorimager scans of ${ }^{35} \mathrm{~S}-\mathrm{methionine-labelled} \mathrm{mitochondrial} \mathrm{translation}$ products separated by SDS-PAGE and blotted to PVDF membranes shown for the indicated cell lines. Subsequent western blotting for GAPDH was performed to ensure equal loading. Approximate size of mitochondrial translation products is indicated to the right of the gel. $\mathbf{d}, \mathrm{Quantification}$ of $n=7$ independent experiments compared with the wild-type sample is plotted with the means (s.d.). ${ }^{\star \star \star} P<0.0001$, unpaired $t$-test, two-tailed. e, Wild-type EXD2, but not the NUC and C2 mutant forms of EXD2, can rescue the translation defect of cells lacking EXD2. f, The mitochondrial translation defect is not rescued by the administration of NAC to the culture medium. Data in e,f represent 1 out of 3 experiments. Controls treated with CAP (to block mitochondrial translation) or CAP without emetine (to allow only cytoplasmic translation) are shown. Additional examples used for quantification are provided in Supplementary Fig. 4, source data and statistics are provided in Supplementary Table 1, and uncropped blots are provided in Supplementary Fig. 9. 

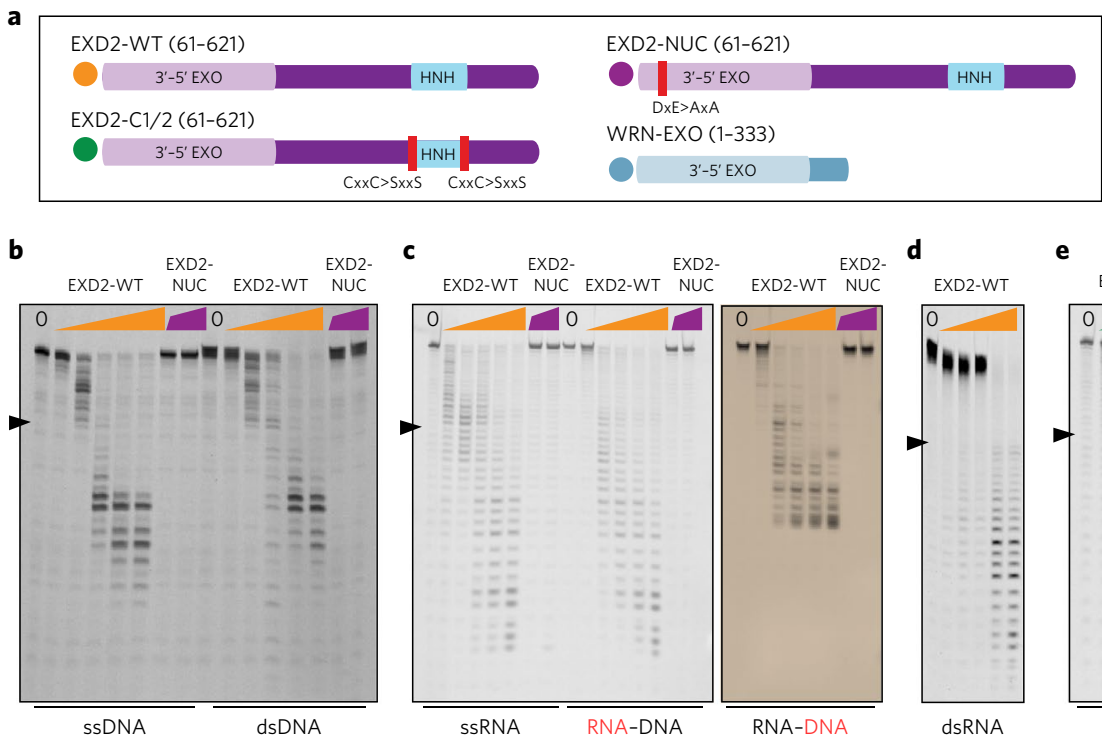

c EXD2-WT NUC EXD2-WT $\begin{aligned} & \text { EXD2- } \\ & \text { NUC }\end{aligned}$ EXD2-WT EXD2d EXD2-WT e
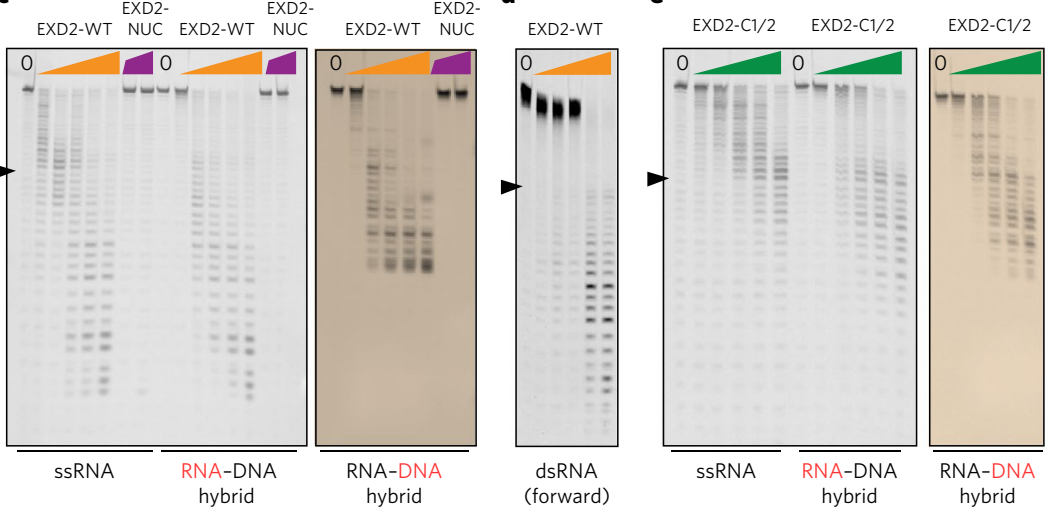

f
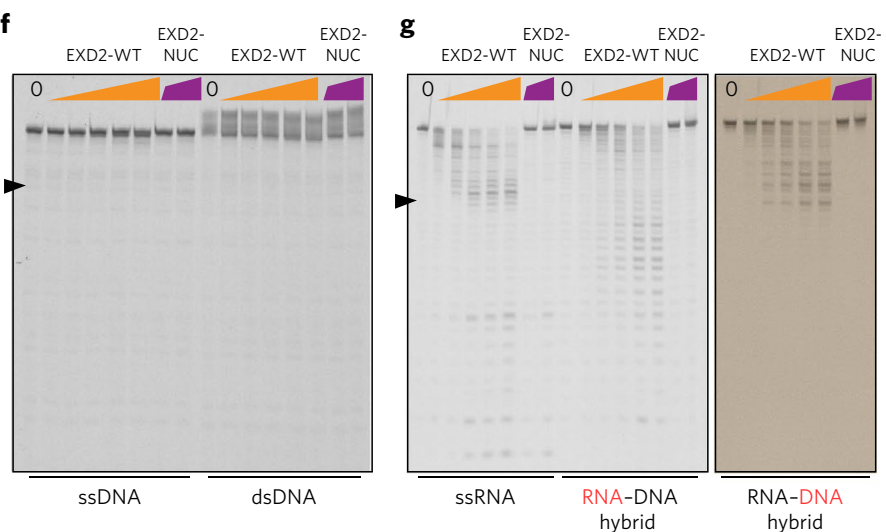

h

i
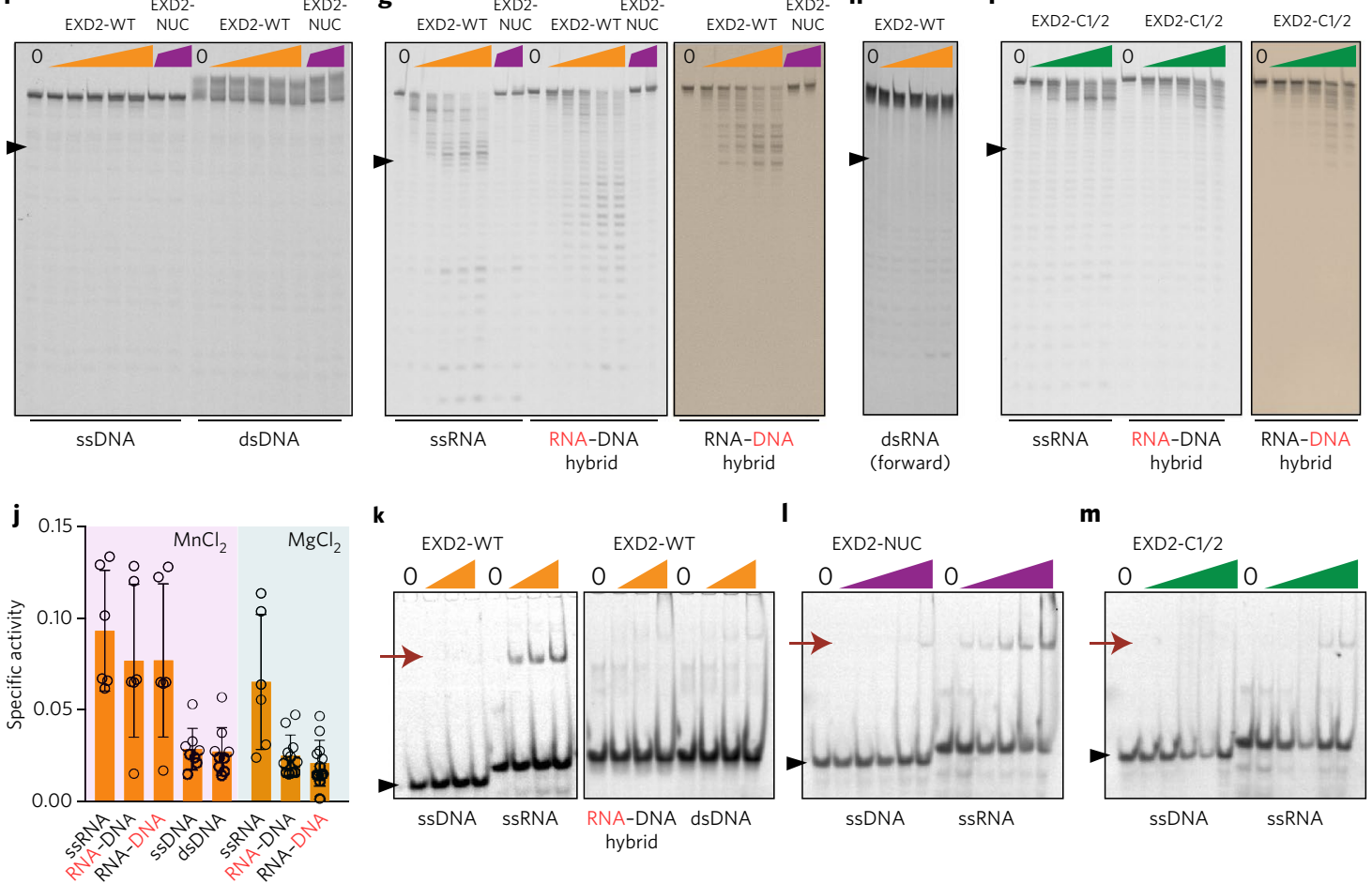

k

I
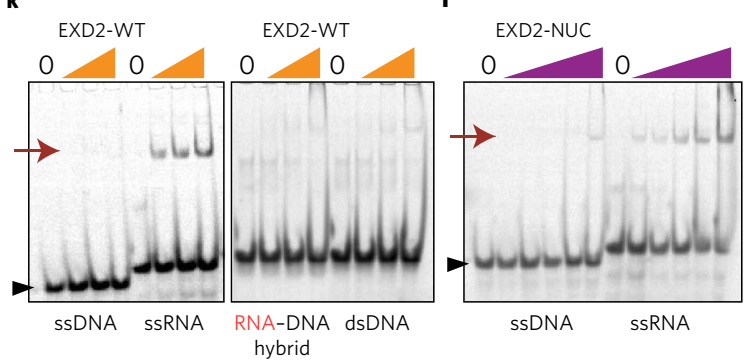

m

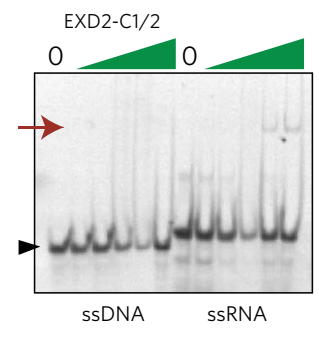

Fig. 6 | EXD2 preferentially targets ssRNA. a, Schematic of proteins used in biochemical analyses. b,c, EXD2-WT (5-80 nM) or EXD2-NUC (40-80 nM) was incubated with $20 \mathrm{nM}$ fluorescently labelled ssDNA or dsDNA (b) or $20 \mathrm{nM}$ RNA-DNA (labelled with FAM or Cy5) hybrid (c) in the presence of $10 \mathrm{mM} \mathrm{MnCl}_{2}$. Red font indicates molecule visualized, and sepia panel is the same gel scanned with a second laser for Cy5-labelled DNA. d, EXD2-WT (5-80 nM) was incubated with a dsRNA FAM substrate (forward) in the presence of $10 \mathrm{mM} \mathrm{MnCl} 2$. e, EXD2-C1/2 (5-80 nM)

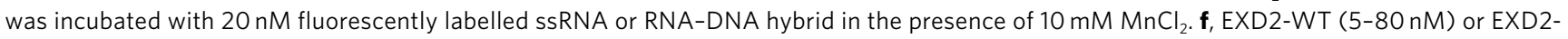
NUC (40-80 nM) was incubated with $20 \mathrm{nM}$ fluorescently labelled ssDNA or dsDNA or $\mathbf{g} 20 \mathrm{nM}$ RNA-DNA (labelled with FAM or Cy5) hybrid in the presence of $10 \mathrm{mM} \mathrm{MgCl}_{2} \cdot \mathbf{h}$, EXD2-WT (5-80 nM) was incubated with a dsRNA FAM substrate (forward) in the presence of $10 \mathrm{mM} \mathrm{MgCl}$. i, EXD2-C1/2 (5-80 nM) was incubated with $20 \mathrm{nM}$ fluorescently labelled ssRNA or RNA-DNA hybrid in the presence of $10 \mathrm{mM} \mathrm{MgCl} 2$. All reactions in b-i were carried out for $30 \mathrm{~min}$ at $37^{\circ} \mathrm{C}$ before being separated on an $18 \%$ polyacrylamide urea TBE gel and 30 bp indicated with black arrowhead. j, Quantification of specific activity (nanomolar substrate cleavage per nanomolar protein per minute) of EXD2-WT on indicated substrates in the presence of $\mathrm{MgCl}_{2}$ or $\mathrm{MnCl}_{2}$. Data compiled from $n=3$ independent experiments and all values with the means (s.d.) are shown. k-m, EXD2-WT $(20-310 \mathrm{nM})(\mathbf{k})$, EXD2-NUC (20-310 nM) (I) and EXD2-C1/2 (20-310 nM) (m) were incubated with $20 \mathrm{nM}$ fluorescently labelled ssDNA, ssRNA, RNA-DNA hybrid or dsDNA for 30 min on ice. Red arrow indicates the protein-nucleic acid complex formed with ssRNA and reduced with the EXD2-C1/2 mutant, and black arrowheads indicate 40 bp. Data in $\mathbf{b}, \mathbf{c}, \mathbf{e}-\mathbf{g}, \mathbf{i}, \mathbf{k}, \mathbf{l}$ are representative of three experiments; data in $\mathbf{d}, \mathbf{h}, \mathbf{m}$ are representative of two experiments. Source data are provided in Supplementary Table 1, and additional analysis and uncropped gels are provided in Supplementary Figs. 5 and 9, respectively. 

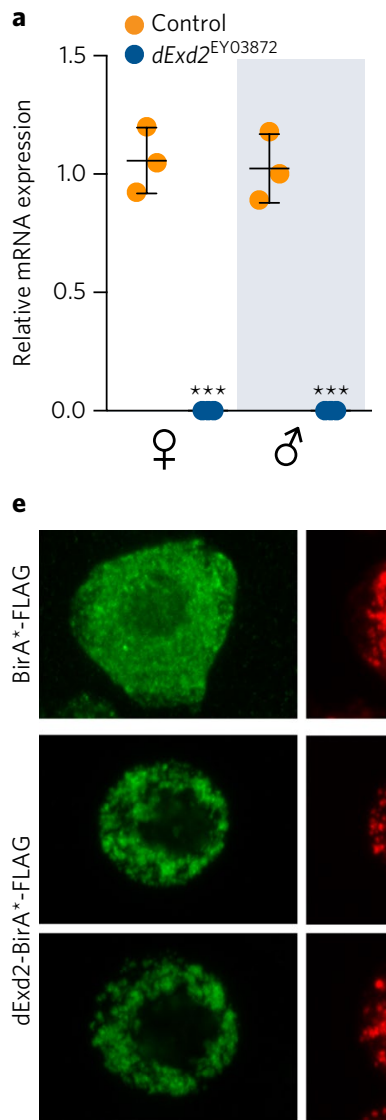

FLAG
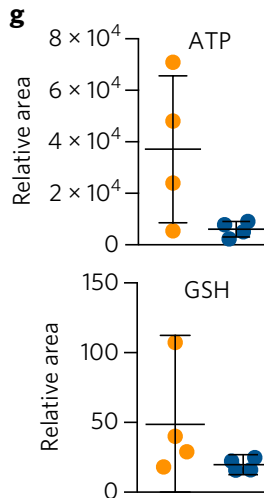

b
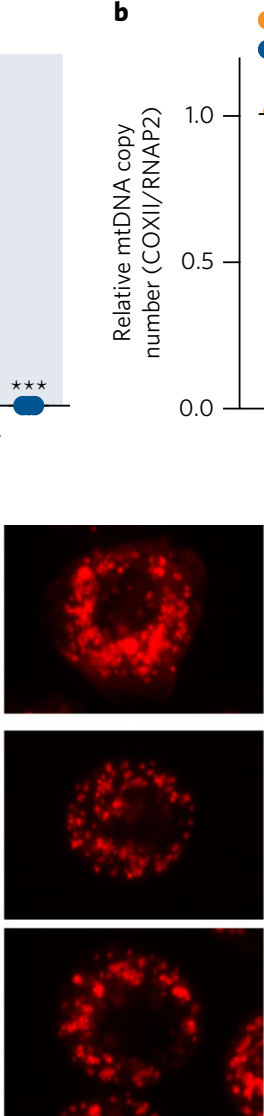

ATP5A
Control

dExd2 $2^{\mathrm{EY} 03872}$

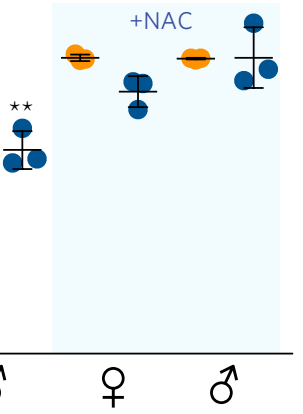

$\underset{\text { Drosohhila S2 cells }}{\widehat{0}}$
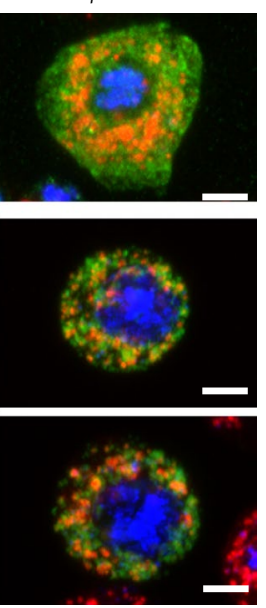

DAPI-merge c

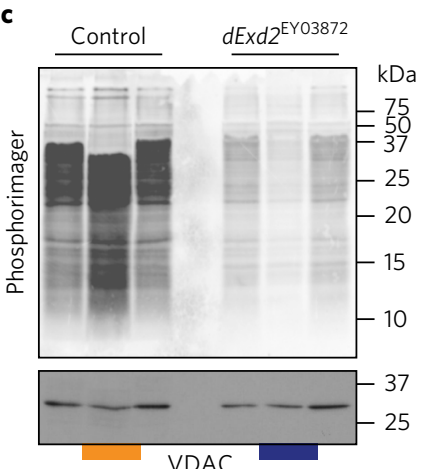

d

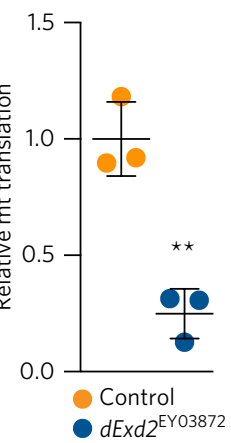

f
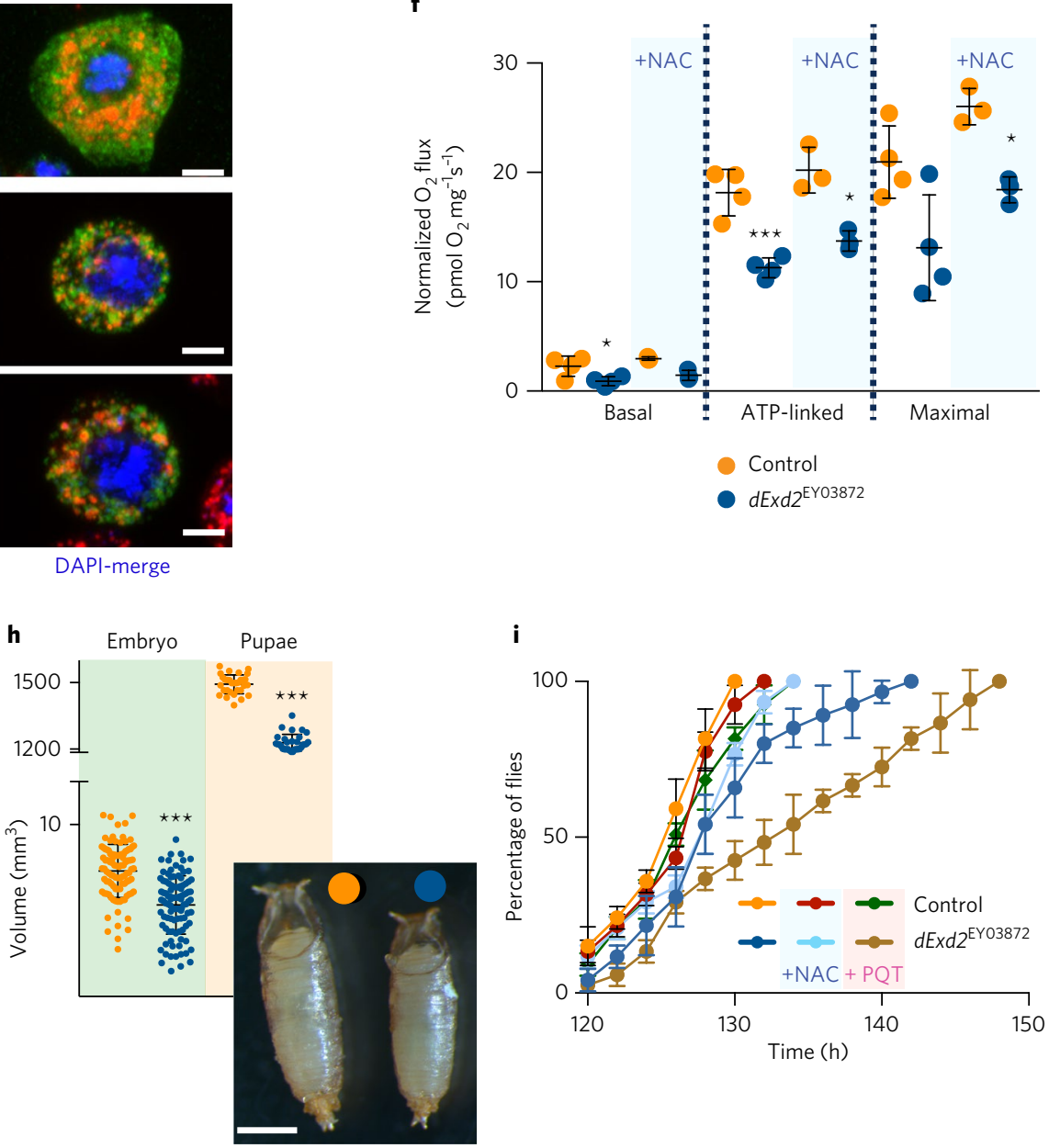

i

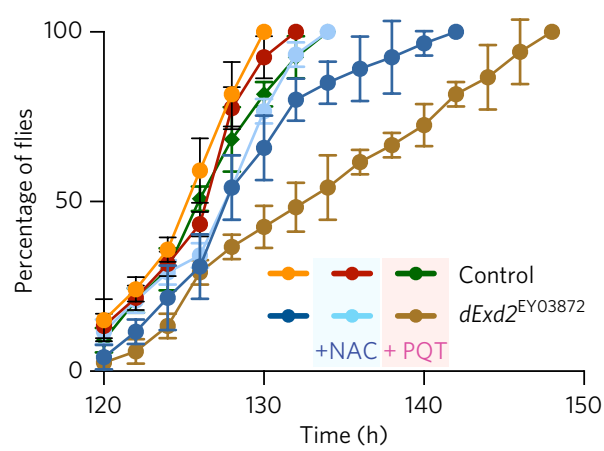

Control

$d E x d 2^{\text {EY03872 }}$

Fig. 7 | Metabolic and developmental defects in dExd2-deficient flies. a, Reduced $d E x d 2$ expression in $d E x d 2^{E Y 03872}$ flies compared with control (yellow white, $y w$ ) measured by qRT-PCR. Averages from $n=3$ independent experiments plotted with the mean ( $\mathrm{s} . \mathrm{d}$.) ( ${ }^{\star \star \star} P=0.0002$ and 0.0003 , unpaired $t$-test, two-tailed). b, mtDNA levels analysed using qRT-PCR in flies reared in food with or without NAC. Averages from $n=3$ experiments plotted with the means (s.d.). ${ }^{\star \star} P=0.0083$ female, ${ }^{\star \star} P=0.0013$ male, unpaired $t$-test, two-tailed. c, Phosphorimager scan of mitochondrial translation products. Western blot for VDAC (porin) shows equal loading. d, Quantification of $n=3$ independent experiments compared with wild-type samples with the means (s.d.) shown with individual values. ${ }^{\star} P=0.0024$, unpaired $t$-test, two-tailed. e, Confocal immunofluorescence of exogenously expressed dEXD2-BirA*-FLAG or BirA*-FLAG protein in Drosophila S2 cells. Extensive colocalization of EXD2-BirA*-FLAG but not BirA*-FLAG is observed with the mitochondrial protein ATP5A. Scale bar, $0.2 \mu \mathrm{m}$. Data represent 30 cells from 2 experiments. f, Reduced oxygen consumption in dEXD2-deficient flies is not rescued with NAC treatment. Measurements from $n=4$ or $n=3$ (NAC samples) independent experiments are plotted with the means (s.d.). ${ }^{\star} P<0.05,{ }^{\star \star \star} P<0.001$, one-way ANOVA with Dunnet's test. $\mathbf{g}, \mathrm{LC}-\mathrm{MS}$ analysis of the indicated metabolites in 1-week-old flies reared on normal food or food containing NAC. $n=4$ independent biological replicates plotted with the means (s.d.). h, $d E x d 2^{E Y 03872}$ embryos and pupae are smaller than controls. Individual values and the means (s.d.) from $n=100$ embryos and $n=31$ pupae are shown ( ${ }^{\star \star \star} P<0.0001, t$-test, two-tailed). A representative example pupa of each genotype is shown; scale bar, $500 \mu \mathrm{m}$. i, Delayed pupariation in $d E x d 2^{\mathrm{EY} 03872} \mathrm{flies}$ is rescued by NAC and enhanced by paraquat (PQT) at doses that do not strongly affect controls. Mean (s.d.) compiled from $n=3$ independent experiments with 40 flies per point is plotted. Source data and additional statistical information are provided in Supplementary Table 1, and uncropped blots are provided in Supplementary Fig. 9. 


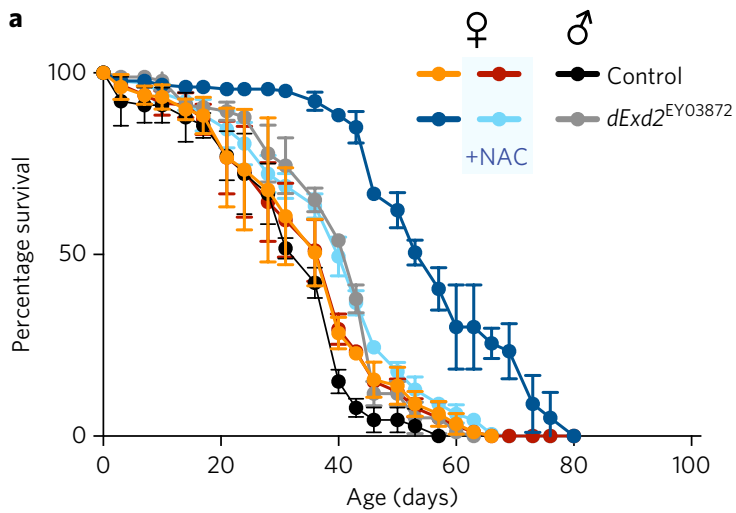

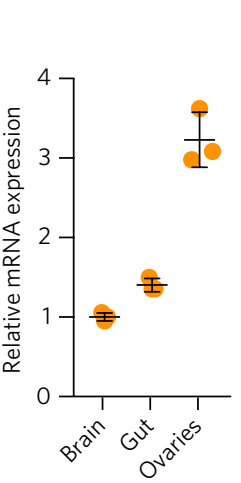

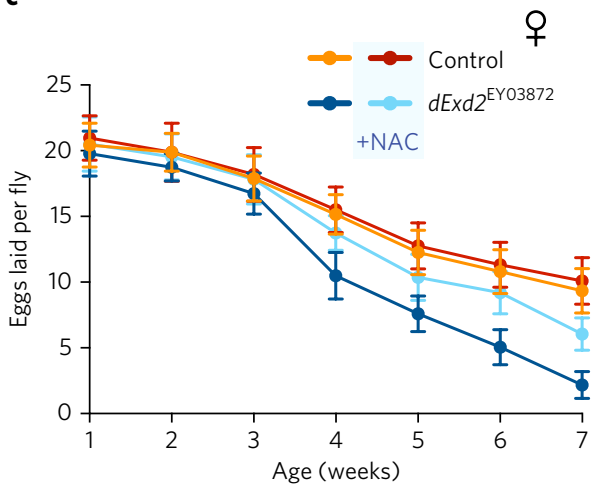

d
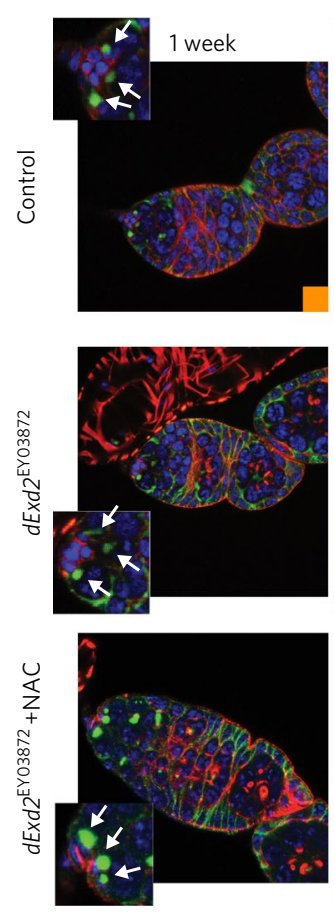
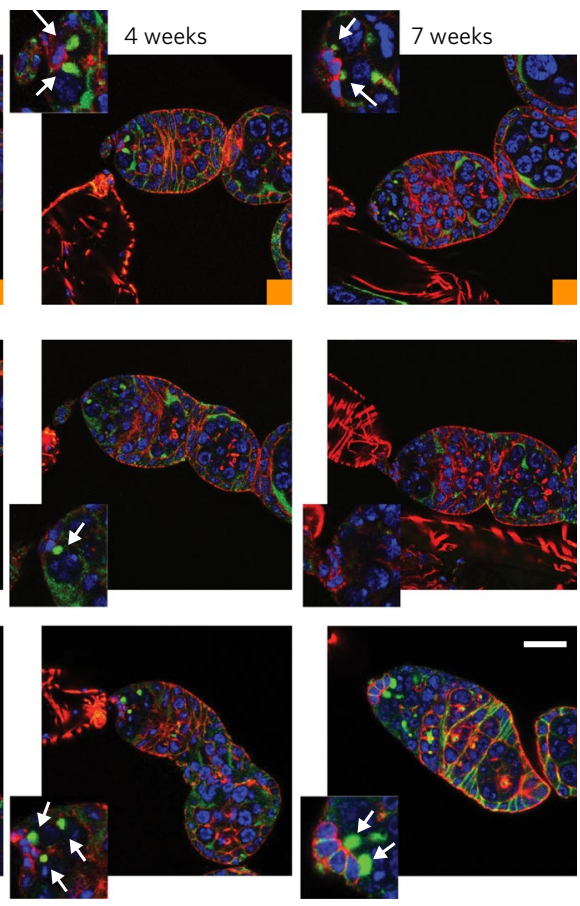

Hts Phalloidin DAPI

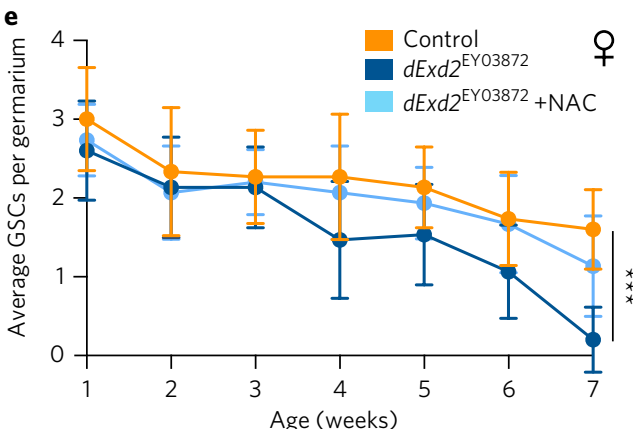

f

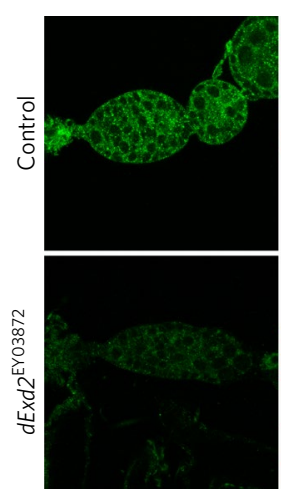

Cyt c

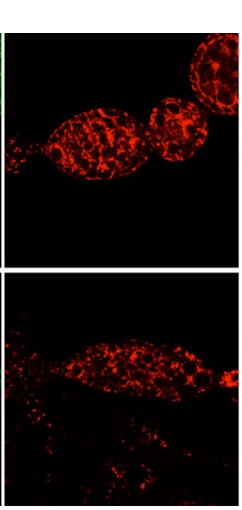

ATP5A

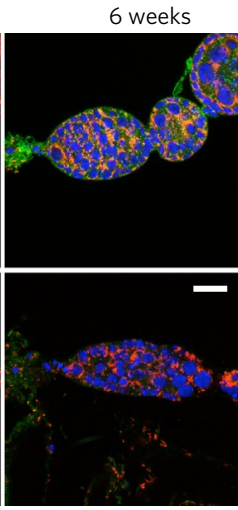

DAPI-merge

Fig. 8 | Germline stem cell attrition and increased lifespan in dEXD2-deficient flies. a, Significantly increased lifespan in dExd2 EY03872 flies compared with controls, particularly for females. NAC normalizes the lifespan of dEXD2-deficient females. Means (s.d.) were compiled from $n=3$ independent experiments, each using 60 flies. ${ }^{\star \star \star} P<4.03 \times 10^{-10}$ for males and ${ }^{\star \star \star} P<2.22 \times 10^{-16}$ for females, proportional hazard Cox model. $\mathbf{b}$, qRT-PCR analysis of different Drosophila tissues shows high $d E x d 2$ mRNA levels in the ovaries of control flies compared with other tissues. Values from $n=3$ independent experiments are plotted with the means (s.d.). c, Fecundity was reduced in $d E x d 2^{\text {EYo3872 }}$ flies and partially rescued by NAC. A total of 60 flies was used for each group and the means (s.d.) shown. d, Age-dependent attrition of GSCs in dExd2 ${ }^{\mathrm{EY} O 3872}$ flies was rescued by NAC. GSCs were identified by Hts staining and indicated by white arrows. Scale bar, $20 \mu \mathrm{m}$. e, Quantification of GSCs of each genotype for the indicated ages with or without NAC treatment. Means were compiled from $n=15$ independent germaria at each time point and the means (s.d.) shown. Statistical analysis was performed using the proportional odds cumulative link model ( ${ }^{\star \star \star} P=1.38 \mathrm{E}-08$ for week 7$)$. $\mathbf{f}$, Immunofluorescence analysis revealed a strong decrease in cytochrome $c$ (Cyt $c$ ) levels and disorganized ATP synthase patterns (ATP5A) in germaria of $d E x d 2^{\mathrm{EY} 03872} \mathrm{flies}$. Scale bar, $20 \mu \mathrm{m}$. Source data and additional statistical analysis are provided in Supplementary Table 1.

We propose that EXD2 functions as a ribonuclease that prevents precocious association of mitochondrial mRNAs with the free $28 \mathrm{~S}$ mitoribosomal subunits before $55 \mathrm{~S}$ assembly. This proposal is consistent with the enrichment of $28 \mathrm{~S}$ proteins observed in the BioID-MS data, the fractionation pattern of EXD2, its preference for ssRNA and the increased mRNA levels co-fractionating with aberrant $28 \mathrm{~S}$ subunits in EXD2-deficient cells. Similar alterations in $28 \mathrm{~S}$ subunit mobility were observed in cells lacking LRPPRC and SLIRP, which influence stability and translation of mitochondrial mRNA pools ${ }^{26,27}$. While we cannot strictly rule out a role for
EXD2 in mtRNA processing or stability, MitoString ${ }^{28}$ analysis indicated that the profiles of EXD2-deficient cells were highly similar to parental or complemented cells, and most known precursor RNA processing events occurred normally (Supplementary Fig. 7a,b), indicating that EXD2 is not likely required for global RNA precursor processing or mitochondrial mRNA levels.

The EXO and HNH-like domains are both crucial for EXD2 function. Extending earlier reports ${ }^{8}$, we identified additional EXD2 orthologues, defined by EXO and HNH domains (Supplementary Fig. 1a-c). Arabidopsis RRP6L3 is a likely EXD2 orthologue with 
a high degree of similarity in the $\mathrm{HNH}$ domain, although its EXO domain is more similar to RRP6 (exosomal RNAse) than WRN and it contains an additional HRDC domain absent in metazoan EXD2 nucleases. EXD2 also appears sporadically in some trypanosome lineages; in Leishmania, EXD2 has an additional Z3H1-type zinc finger not present in other species. Thus, EXD2 is widely conserved and at least three major domain configurations exist, potentially reflecting a specialization of function in those organisms.

Our data suggest that the HNH-like motif influences stable binding of EXD2 to ssRNA and the $28 \mathrm{~S}$ subunit (Figs. 4,5), reflected by the absence of EXD2-SF-C2 in 28S fractions (Fig. 5a) and its reduced RNA binding. The EXD2 $\mathrm{HNH}$-like domain lacks conserved DH catalytic residues required for activity in other $\mathrm{HNH}$ endonucleases (Supplementary Fig. 1d). Notably, in fold and function assignment searches (FFAS), several of the highest scoring $\mathrm{HNH}$ domains belong to proteins involved in chaperoning assembly and insertion of membrane complexes (DotN) or electron transport (NapB), suggesting a diverse repertoire of potential functions for this domain (Supplementary Fig. 1d).

Both human and Drosophila EXD2 were identified in a genomewide short interfering RNA screen for host factors that support dengue RNA virus replication; however, a specific role in the viral lifecycle has not been reported ${ }^{29}$. EXD2 was also implicated in DNA repair, reported to localize to nuclear chromatin and interact with the DNA resection machinery, and identified on collapsed replication forks in an isolation of proteins on nascent DNA(iPOND)-MS screen $^{7-10,30}$. However, 11 mitochondrial proteins, including 5 mitoribosome proteins, were also identified in the iPOND-MS screen ${ }^{30}$ with comparable scores. Using reported fractionation methods ${ }^{8}$, we readily identify EXD2 in chromatin fractions (Supplementary Fig. 7c), but they were highly contaminated with mitochondria, and these points should be considered in light of the data reported here. We did not detect EXD2 in the nucleus (Fig. 1 and Supplementary Fig. 1h), and overexpressed human and fly EXD2 localized primarily to mitochondria with no clear nuclear staining in immunofluorescence assays (Figs. 1, 4 and 7). This result is consistent also with the cytoplasmic localization reported for Arabidopsis RRP6L $3^{31}$. Moreover, in an unbiased proteomics approach, we identified almost exclusively mitochondrial proteins, none of which has been implicated in DNA repair or replication, except for POLD4 (POLDIP2) and ATR, which also have mitochondrial $\operatorname{roles}^{32,33}$ (Fig. 4c and Supplementary Table 2).

Consistent with previous reports ${ }^{8}$, we observed robust $3^{\prime}-5^{\prime}$ exonuclease activity, but both EXD2 and WRN showed activity on all DNA and RNA substrates tested (Fig. 6 and Supplementary Fig. 5) when $\mathrm{MnCl}_{2}$ was used in reactions. In contrast, EXD2 showed clear binding and activity on ssRNA in the presence of $\mathrm{MgCl}_{2}$, suggesting that its physiological substrate is RNA. Additionally, we did not observe defective RPA focus formation (Supplementary Fig. 7d,e) or DNA damage sensitivity in EXD2-deficient cells using several cell line backgrounds controlled with complementation (Supplementary Fig. $7 \mathrm{f}-\mathrm{h}$ ). We believe that our data strongly indicates that the primary role of EXD2 is to govern mitochondrial ribosome mRNA loading, translation and ATP production through its RNAse and potentially other activities. However, we cannot rule out a DNA replication or repair role that we have not detected owing to differences in technical conditions or cell line backgrounds. Notably, phenotypes associated with some translational regulators include defects in mitochondrial and nuclear genome maintenance ${ }^{34}$, and there are examples of DNA repair enzyme families with key roles in translational regulation ${ }^{35}$. Moreover, numerous DNA repair nucleases (for example, FEN1 and DNA2) localize to both the nucleus and mitochondria ${ }^{36,37}$.

ROS levels, reproductive capacity, abundance and activity of OXPHOS components and global translation have all been shown to be major factors influencing lifespan in numerous organisms ${ }^{4,6,38-40}$. Depletion of mitochondrial translational regulators in Saccharomyces cerevisae extends lifespan in a Sir2-dependent manner ${ }^{41}$, but we did not observe alterations in Sir2 mRNA in dEXD2-deficient flies, and lifespan extension in females was independent of calorie restriction, which is also Sir2 dependent ${ }^{42}$ (Supplementary Fig. 7i,j). We propose that the dramatic lifespan extension in dEXD2-deficient females is primarily the result of ROS-mediated, premature attrition of the GSC pool and loss of fecundity (Supplementary Fig. 6e). This proposal is consistent with $d E x d 2$ expression patterns, the rescue of GSC attrition, fecundity and lifespan with NAC treatment, the reduced respiration observed in $\mathrm{dEXD} 2$-deficient flies, and the close correlation between the age of GSC loss and lifespan extension (Fig. 8). An inverse relationship between lifespan and reproductive capacity was reported in several organisms, including Caenorhabditis elegans and Drosophila ${ }^{25}$. Although multiple mechanisms have been proposed, including elevated ROS and antagonistic GSC to soma crosstalk, the precise nature of the signal that influences lifespan remains to be clarified ${ }^{43}$.

While it remains unclear whether EXD2 influences mammalian fertility or lifespan, analysis of mRNAs enriched in Xenopus germ plasm, which is rich in mitochondria and RNA-binding proteins, identified $\mathrm{xEXD} 2{ }^{44}$; this finding indicates that $\mathrm{xEXD} 2$ could play a similar role in the vertebrate GSC niche. Patients with interstitial deletions in chromosome 14q24, which includes EXD2 and 18 neighboring genes, have been reported to have congenital heart defects, brachydactyly and intellectual disability ${ }^{45}$, indicating that EXD2 loss may influence human development. However, the individual contribution of EXD2 deficiency to these pathologies remains unclear. The precise control of mtDNA levels, RNA processing, ribosome assembly, translation, and the dynamic composition and molecular regulation of OXPHOS complexes in mammals is critical for normal metabolism and healthy ageing ${ }^{1}$. Our results, and those of others, suggest that many additional regulators of these processes have yet to be identified, and their characterization will further enhance our understanding of mitochondrial regulation and its role in ageing.

\section{Methods}

Methods, including statements of data availability and any associated accession codes and references, are available at https://doi. org/10.1038/s41556-017-0016-9.

Received: 10 November 2016; Accepted: 27 November 2017; Published online: 15 January 2018

\section{References}

1. Kauppila, T. E., Kauppila, J. H. \& Larsson, N. G. Mammalian mitochondria and aging: an update. Cell Metab. 25, 57-71 (2017).

2. Jourdain, A. A., Boehm, E., Maundrell, K. \& Martinou, J. C. Mitochondrial RNA granules: compartmentalizing mitochondrial gene expression. J. Cell Biol. 212, 611-614 (2016).

3. Chinnery, P. F. Mitochondrial disease in adults: what's old and what's new? EMBO Mol. Med. 7, 1503-1512 (2015).

4. Yang, W. \& Hekimi, S. Two modes of mitochondrial dysfunction lead independently to lifespan extension in Caenorhabditis elegans. Aging Cell 9 , 433-447 (2010)

5. Schmeisser, S. et al. Neuronal ROS signaling rather than AMPK/sirtuin mediated energy sensing links dietary restriction to lifespan extension. Mol. Metab. 2, 92-102 (2013).

6. Dillin, A. et al. Rates of behavior and aging specified by mitochondrial function during development. Science 298, 2398-2401 (2002).

7. Smogorzewska, A. et al. A genetic screen identifies FAN1, a Fanconi anemia-associated nuclease necessary for DNA interstrand crosslink repair. Mol. Cell 39, 36-47 (2010)

8. Broderick, R. et al. EXD2 promotes homologous recombination by facilitating DNA end resection. Nat. Cell Biol. 18, 271-280 (2016).

9. Cox, L. S., Clancy, D. J., Boubriak, I. \& Saunders, R. D. Modeling Werner syndrome in Drosophila melanogaster: hyper-recombination in flies lacking WRN-like exonuclease. Ann. NY Acad. Sci. 1119, 274-288 (2007). 
10. Biehs, R. et al. DNA double-strand break resection occurs during nonhomologous end joining in G1 but is distinct from resection during homologous recombination. Mol. Cell 65, 671-684.e5 (2017).

11. Kudlow, B. A., Kennedy, B. K. \& Monnat, R. J. Jr. Werner and HutchinsonGilford progeria syndromes: mechanistic basis of human progeroid diseases Nat. Rev. Mol. Cell Biol. 8, 394-404 (2007).

12. Evans, A. M., DeHaven, C. D., Barrett, T., Mitchell, M. \& Milgram, E. Integrated, nontargeted ultrahigh performance liquid chromatography/ electrospray ionization tandem mass spectrometry platform for the identification and relative quantification of the small-molecule complement of biological systems. Anal. Chem. 81, 6656-6667 (2009).

13. Vinaixa, M. et al. Positional enrichment by proton analysis (PEPA): a one-dimensional ${ }^{1} \mathrm{H}$-NMR approach for ${ }^{13} \mathrm{C}$ stable isotope tracer studies in metabolomics. Angew. Chem. Int. Ed. Engl. 56, 3531-3535 (2017).

14. Deberardinis, R. J., Sayed, N., Ditsworth, D. \& Thompson, C. B. Brick by brick: metabolism and tumor cell growth. Curr. Opin. Genet. Dev. 18 54-61 (2008).

15. Cong, L. et al. Multiplex genome engineering using CRISPR/Cas systems. Science 339, 819-823 (2013)

16. Fukuoh, A. et al. Screen for mitochondrial DNA copy number maintenance genes reveals essential role for ATP synthase. Mol. Syst. Biol. 10, 734 (2014)

17. West, A. P., Shadel, G. S. \& Ghosh, S. Mitochondria in innate immune responses. Nat. Rev. Immunol. 11, 389-402 (2011).

18. Smeitink, J. A., Zeviani, M., Turnbull, D. M. \& Jacobs, H. T. Mitochondrial medicine: a metabolic perspective on the pathology of oxidative phosphorylation disorders. Cell Metab. 3, 9-13 (2006).

19. Roux, K. J., Kim, D. I. \& Burke, B. BioID: a screen for protein-protein interactions. Curr. Protoc. Protein Sci. 74, 19.23.1-19.23.4 (2013).

20. Sanchez-Caballero, L., Guerrero-Castillo, S. \& Nijtmans, L. Unraveling the complexity of mitochondrial complex I assembly: a dynamic process. Biochim. Biophys. Acta 1857, 980-990 (2016).

21. Sasarman, F. \& Shoubridge, E. A. Radioactive labeling of mitochondrial translation products in cultured cells. Methods Mol. Biol. 837, 207-217 (2012).

22. Attrill, H. et al. FlyBase: establishing a Gene Group resource for Drosophila melanogaster. Nucleic Acids Res. 44, D786-D792 (2016).

23. Merkey, A. B., Wong, C. K., Hoshizaki, D. K. \& Gibbs, A. G. Energetics of metamorphosis in Drosophila melanogaster. J. Insect Physiol. 57, 1437-1445 (2011).

24. Hsu, H. J. \& Drummond-Barbosa, D. Insulin levels control female germline stem cell maintenance via the niche in Drosophila. Proc. Natl Acad. Sci. USA 106, 1117-1121 (2009)

25. Hansen, M., Flatt, T. \& Aguilaniu, H. Reproduction, fat metabolism, and life span: what is the connection? Cell Metab. 17, 10-19 (2013).

26. Ruzzenente, B. et al. LRPPRC is necessary for polyadenylation and coordination of translation of mitochondrial mRNAs. EMBO J. 31, 443-456 (2012).

27. Bratic, A. et al. The bicoid stability factor controls polyadenylation and expression of specific mitochondrial mRNAs in Drosophila melanogaster. PLoS Genet. 7, e1002324 (2011).

28. Wolf, A. R. \& Mootha, V. K. Functional genomic analysis of human mitochondrial RNA processing. Cell Rep. 7, 918-931 (2014).

29. Sessions, O. M. et al. Discovery of insect and human dengue virus host factors. Nature 458, 1047-1050 (2009).

30. Sirbu, B. M. et al. Identification of proteins at active, stalled, and collapsed replication forks using isolation of proteins on nascent DNA (iPOND) coupled with mass spectrometry. J. Biol. Chem. 288, 31458-31467 (2013).

31. Lange, $H$. et al. Degradation of a polyadenylated rRNA maturation by-product involves one of the three RRP6-like proteins in Arabidopsis thaliana. Mol. Cell Biol. 28, 3038-3044 (2008).

32. Xie, B. et al. Further characterization of human DNA polymerase delta interacting protein 38. J. Biol. Chem. 280, 22375-22384 (2005).

33. Hilton, B. A. et al. ATR plays a direct antiapoptotic role at mitochondria, which is regulated by prolyl isomerase Pin 1. Mol. Cell 60, 35-46 (2015).

34. Oberto, J. et al. Qri7/OSGEPL, the mitochondrial version of the universal $\mathrm{Kael} /$ YgjD protein, is essential for mitochondrial genome maintenance. Nucleic Acids Res. 37, 5343-5352 (2009).

35. Ougland, R., Rognes, T., Klungland, A. \& Larsen, E. Non-homologous functions of the AlkB homologs. J. Mol. Cell Biol. 7, 494-504 (2015).

36. Liu, P. et al. Removal of oxidative DNA damage via FEN1-dependent long-patch base excision repair in human cell mitochondria. Mol. Cell Biol. 28, 4975-4987 (2008).
37. Zheng, L. et al. Human DNA2 is a mitochondrial nuclease/helicase for efficient processing of DNA replication and repair intermediates. Mol. Cell 32, 325-336 (2008)

38. Copeland, J. M. et al. Extension of Drosophila life span by RNAi of the mitochondrial respiratory chain. Curr. Biol. 19, 1591-1598 (2009).

39. Miwa, S. et al. Low abundance of the matrix arm of complex I in mitochondria predicts longevity in mice. Nat. Commun. $\mathbf{5}$, 3837 (2014).

40. Scialo, F. et al. Mitochondrial ROS produced via reverse electron transport extend animal lifespan. Cell Metab. 23, 725-734 (2016).

41. Caballero, A. et al. Absence of mitochondrial translation control proteins extends life span by activating sirtuin-dependent silencing. Mol. Cell 42, 390-400 (2011)

42. Guarente, L. \& Picard, F. Calorie restriction-the SIR2 connection. Cell 120, 473-482 (2005)

43. Quiros, P. M., Mottis, A. \& Auwerx, J. Mitonuclear communication in homeostasis and stress. Nat. Rev. Mol. Cell Biol. 17, 213-226 (2016).

44. Cuykendall, T. N. \& Houston, D. W. Identification of germ plasm-associated transcripts by microarray analysis of Xenopus vegetal cortex RNA. Dev. Dyn. 239, 1838-1848 (2010).

45. Oehl-Jaschkowitz, B. et al. Deletions in 14q24.1q24.3 are associated with congenital heart defects, brachydactyly, and mild intellectual disability. Am. J. Med. Genet. A 164A, 620-626 (2014).

\section{Acknowledgements}

We are grateful to A. Zorzano, A. Vaquero, E. Hidalgo, W. M. Keyes, M. Milan, J. Casanova, M. Solà, V. Mootha, J. Guinovart and the Stracker Lab for input and discussions, N. Gallisa for help generating BioID constructs, J. J. P. Perry and J.A. Tainer for the WRN-EXO expression construct, A. Bratic and N.G. Larsson for sharing advice and protocols, and the Advanced Digital Microscopy and Biostatistics/Bioinformatics IRB core facilities. We thank the following bodies for funding: Ministerio de Economía y Competitividad (MINECO) (T.H.S.: BFU2012-39521, BFU2015-68354, Ayudas para incentivar la incorporación estable de doctores (IED) 2015; L.R.d.P.: BIO2015-64572; T.H.S. and L.R.d.P: institutional funding through the Centres of Excellence Severo Ochoa award and from the CERCA Programme of the Catalan Government; and O.Y.: SAF2011-30578 and BFU2014-57466); the Spanish Biomedical Research Centre in Diabetes and Associated Metabolic Disorders (CIBERDEM), an initiative of Instituto de Investigacion Carlos III (ISCIII) to O.Y.; and the Biotechnology and Biological Sciences Research Council (BBSRC: BB/H019723/1 and BB/M008800/1) to A.J.D. and L.B. S.A. was supported by a Finnish Cultural Society Fellowship, J.S. by a fellowship from Fundação para a Ciência e a Tecnologia (SFRH/BD/87025/2012), P.A.K. by an Advanced Postdoc Mobility fellowship from the Swiss National Science Foundation (SNF), I.G.-C. by an Asociación Española Contra el Cáncer (AECC) fellowship, A.A.-F. by a Severo Ochoa FPI fellowship (MINECO; SVP2014068398), and A.A.J. by an EMBO long-term fellowship (ALTF 554-2015).

\section{Author contributions}

J.S. and S.A. performed the majority of experiments characterizing cell lines and effects of EXD2 deficiency. J.S. performed sucrose gradient fractionations, and L.J.B. and A.J.D. purified and characterized bacterial EXD2 and WRN, performed mtDNA replication assays and analysed data. J.S., S.A., A.C. and A.G.-R. characterized Drosophila. P.A.K. and P.P.-F. performed BioID assays, and M.V., S.S.-G. and O.Y. carried out metabolomics and mass spectrometry, analysed data and prepared figures. E.C. and B.R. purified biotinylated peptides, performed mass spectrometry and analysed data. A.A.J. performed the MitoString assay, and I.G.-C., J.S., S.A. and T.H.S. performed survival assays and analysed DNA damage responses. T.H.S. and J.S. performed mitochondrial translation assays, and P.A.K. and A.A.F. performed immunofluorescence analysis in Drosophila S2 cells. A.M.R. performed computational and evolutionary analysis, and L.R.d.P. provided critical technical expertise and advice. T.H.S. analysed data, prepared figures, supervised experiments and wrote the manuscript with editorial contributions from all authors.

\section{Competing interests}

The authors declare no competing financial interests.

\section{Additional information}

Supplementary information is available for this paper at https://doi.org/10.1038/ s41556-017-0016-9.

Reprints and permissions information is available at www.nature.com/reprints.

Correspondence and requests for materials should be addressed to T.H.S.

Publisher's note: Springer Nature remains neutral with regard to jurisdictional claims in published maps and institutional affiliations. 


\section{Methods}

Generation of expression constructs. Human EXD2 was amplified from U2OS cell complementary DNA, sequenced and cloned into pENTR/D-TOPO (Invitrogen) following the manufacturer's protocol. EXD2 was subcloned using LR clonase into the retroviral vector pLPC-C-SF-TAP generated by subcloning the C-SF-TAP cassette (a gift from C. J. Gloeckner, University of Tübingen ${ }^{46}$ ) into the pLPC-N-Myc plasmid (a gift from T. de Lange; Addgene, plasmid number 12540). EXD2 mutants were generated using a QuikChange Lightning Site-Directed Mutagenesis kit (Agilent Technologies, 210518-5) according to the manufacturer's instructions. Clones were purified using a PureLink Quick Plasmid Miniprep kit (Invitrogen) and the sequence of constructs confirmed. Large-scale plasmid purification was performed using a PureYield Plasmid Maxiprep System (Promega). Primers sequences are provided in Supplementary Table 3.

Human cell culture. Human cell lines (U2OS, HEK293T, AD293, MDAMB-231 and RPE1) were cultured in Dulbecco's modified Eagle Medium (DMEM; Invitrogen, 41966) supplemented with 10\% (v/v) fetal bovine serum (FBS; Sigma, F7524-500ML) and $100 \mathrm{U} \mathrm{ml}^{-1}$ penicillin-streptomycin (Reactiva, $01030311 \mathrm{~B} 000)$ in $5 \% \mathrm{CO}_{2}$. In some experiments, cells were treated with $100 \mu \mathrm{g} \mathrm{ml}^{-1}$ chloramphenicol (CAP) (Sigma, C3175), $25 \mu$ M FCCP (Sigma, C2920) or $5 \mathrm{mM}$ NAC (Sigma, A9165). Cells were periodically tested for mycoplasma using PCR (primers provided in Supplementary Table 3) and results were negative.

Generation of retrovirus and lentivirus and stable cell lines. For viral production, HEK293T cells were seeded in 10 -cm plates $24 \mathrm{~h}$ before transfection with polyethyleneimide (PEI; Polysciences Euro). For lentivirus, a mixture of $10 \mu \mathrm{g}$ lentivirus-short hairpin RNA, $2 \mu \mathrm{g}$ REV, $6 \mu \mathrm{g}$ RSV-RRE and $2 \mu \mathrm{g}$ VSV-G plasmids ${ }^{47}$ with $78 \mu \mathrm{l} \mathrm{PEI}$ and $900 \mu \mathrm{l} 150 \mathrm{mM} \mathrm{NaCl}$ was added to the cell culture media. For retrovirus, a mixture of $10 \mu \mathrm{g}$ retroviral vector DNA, $9 \mu \mathrm{g}$ GAG-Pol (pCL-Eco) ${ }^{48}$ and $1 \mu \mathrm{g}$ VSV-G plasmids with $78 \mu \mathrm{l}$ PEI and $900 \mu \mathrm{l} 150 \mathrm{mM} \mathrm{NaCl}$ was added to the cell culture media. In both cases, $16 \mathrm{~h}$ later, cells were washed in PBS and the media replaced. After $48 \mathrm{~h}$, the viral supernatant was removed, cleared with a $0.45 \mu \mathrm{m}$ filter and incubated with target cells. Three days after infection, cells were selected with puromycin (Sigma, P8833-25MG) for 5 days and subsequently maintained in normal growth medium. Lentivirus shRNA constructs were purchased from the Sigma MISSION lentiviral library against EXD2 (sh31: TRCN0000051631 (NM_018199.1155s1c1); sh22: TRCN0000327822 (NM_018199.2-1001s21c1)), and shRNA against GFP (SCH005) and sh-non-targeter (shNT, SHC016) were used as controls.

Generation of EXD2-KO cells with CRISPR-Cas9. Forward and reverse oligonucleotides containing the guide sequence were annealed and cloned into pX330-U6-Chimeric_BB-CBh-hSpCas9 (a gift from F. Zhang; Addgene plasmid no. 42230) $)^{15}$ modified to coexpress enhanced GFP (a gift from C. Cortina and E. Batlle), and this construct was transfected into U2OS and MDA-MB-231 cells using Lipofectamine 2000 Transfection Reagent (ThermoFisher). GFP-positive cells were sorted $24 \mathrm{~h}$ after transfection (BD FACSAria III SORP) and expanded from single cells. Screening for EXD2-KO cells was carried out by western blotting. Two clones each of U2OS and MDA-MB-231 showing complete loss of EXD2 protein were selected for further phenotypic analysis.

Isolation of DNA, RNA and cDNA preparation. For DNA isolation of human cancer cell lines, 1,000,000 cells were plated on $10 \mathrm{~cm}$ plates and collected $24 \mathrm{~h}$ later in $515 \mu \mathrm{l}$ of lysis buffer ( $75 \mathrm{mM} \mathrm{NaCl}, 50 \mathrm{mM}$ EDTA, $0.02 \%$ SDS, $\left.0.4 \mathrm{mg} \mathrm{ml}^{-1} \mathrm{PK}\right)$. Lysate was transferred to $1.5 \mathrm{ml}$ Eppendorf tubes and incubated at $50^{\circ} \mathrm{C}$ for $2 \mathrm{~h}$. Isopropanol (1 volume) was added and mixed and incubated at $4^{\circ} \mathrm{C}$ overnight. Tubes were centrifuged at $8,500 \mathrm{~g}$ for $30 \mathrm{~min}$ at $4{ }^{\circ} \mathrm{C}$ and pellets washed with $70 \%$ ethanol and air dried. DNA was resuspended in TE buffer and diluted to $100 \mathrm{ng} \mathrm{ml}^{-1}$ for quantitative PCR. D. melanogaster DNA was obtained from adult flies that were snap frozen in liquid nitrogen and the abdomens homogenized with a pestle in grinding buffer $(0.2 \mathrm{M}$ sucrose, $0.1 \mathrm{M}$ Tris buffer $\mathrm{pH} 9.2,50 \mathrm{mM}$ EDTA and $0.5 \%$ SDS ) and incubated at $65^{\circ} \mathrm{C}$ for $10 \mathrm{~min}$. Potassium acetate was added to a final concentration of $5 \mathrm{M}$ and the samples were incubated on ice for $20 \mathrm{~min}$. DNA was precipitated with phenol:chloroform:ethanol. Total RNA was isolated using a PureLink RNA mini kit (Ambion) according to the manufacturer's instructions. Total RNA $(1 \mu \mathrm{g})$ was used as a template for cDNA synthesis using a High Capacity RNA-to-cDNA kit (ThermoFisher).

Quantitative RT-PCR. Quantitative RT-PCR (qRT-PCR) was performed using a Step-One-Plus real-time PCR instrument (Applied Biosystems) and quantified using the comparative CT method. Amplification was performed using Power SYBR Green PCR master mix (Applied Biosystems, 4309155). Analysis of EXD2 mRNA levels was performed using Taqman probes from Applied Biosystems (EXD2 Hs00217045_m1, ACTB 4352935E) together with Taqman master mix (Applied Biosystems). Primers used are provided in Supplementary Table 3.

Flow cytometry-based assays for mitochondrial function. For the analysis of mitochondrial mass, 150,000 cells were seeded in 6-well plates in duplicate $24 \mathrm{~h}$ before analysis. Cells were incubated for $30 \mathrm{~min}$ with $250 \mathrm{nM}$ MitoTracker Green FM or Deep Red probes (ThermoFisher) in normal growth medium at $37^{\circ} \mathrm{C}$.
Cells were trypsinized and washed with warm PBS once, stained with $500 \mathrm{nM}$ 4',6-diamidino-2-phenylindole, dihydrochloride (DAPI) and immediately analysed by flow cytometry. To detect intracellular and mitochondrial ROS production, 150,000 cells were seeded in 6-well plates in duplicate $24 \mathrm{~h}$ before analysis. Cells were incubated at $37^{\circ} \mathrm{C}$ for $30 \mathrm{~min}$ in $5 \mu \mathrm{M}$ MitoSox (Molecular Probes, M36008) and then trypsinized and washed with warm PBS once and analysed immediately by flow cytometry. Data were collected with a Gallios flow cytometer using Kaluza for Gallios software (Beckman Coulter) and analysed using FlowJo v9.9.6 (FlowJo LLC). Data were plotted and statistically analysed in Prism 7, and examples provided in Supplementary Fig. 8.

Immunofluorescence. For immunofluorescence, cells were fixed with $4 \%$ formaldehyde for $10 \mathrm{~min}$ at room temperature. After two washes with PBS, cells were permeabilized with PBS containing 2\% Triton X-100 for $10 \mathrm{~min}$ at room temperature. Cells were washed twice with PBS and incubated with primary antibody (1:500) in PBS-T containing $10 \% \mathrm{FBS}$ for $1 \mathrm{~h}$ at room temperature. After two washes with PBS-T, cells were incubated with secondary antibody (1:500) in PBS-T containing 10\% FBS and DAPI for $1 \mathrm{~h}$ at room temperature. Slides were rinsed in PBS-T, washed twice for $5 \mathrm{~min}$ in PBS-T and mounted with Vectashield (Vector Laboratories, H-1000). Drosophila embryos $(0-15 \mathrm{~h})$ were collected, dechorionated with bleach for $90 \mathrm{~s}$ and rinsed with $0.1 \%$ Triton X-100. Embryos were incubated in a solution containing $4 \%$ formaldehyde and heptane (1:1) for $20 \mathrm{~min}$ in a rotator at room temperature. Formaldehyde was replaced with methanol, samples were vortexed for $20 \mathrm{~s}$ and the embryos were transferred to new vials and washed extensively with PBS-T containing $0.3 \%$ bovine serum albumin (BSA). Samples were incubated with primary antibody for $4 \mathrm{~h}$ at room temperature, and after two washes with PBS-T they were incubated with secondary antibody for $2 \mathrm{~h}$ at room temperature, followed by an incubation with DAPI for $15 \mathrm{~min}$ at room temperature. Embryos were rinsed in PBS-T, washed twice for $5 \mathrm{~min}$ in PBS-T containing $0.3 \%$ BSA and mounted with Fluoromount (Sigma, F4680). Adult ovaries from Drosophila were dissected in Schneider medium and fixed in $4 \%$ formaldehyde in PBS for $20 \mathrm{~min}$ at room temperature. After washing with PBS, they were permeabilized with $1 \%$ Triton X-100 in PBS for $2 \mathrm{~h}$ and then incubated in PBS-T ( $1 \%(\mathrm{w} / \mathrm{v})$ BSA, $0.2 \%$ Triton X-100, PBS) for $1 \mathrm{~h}$. Ovaries were then incubated with primary antibodies diluted in PBS-T overnight at $4^{\circ} \mathrm{C}$. The next day, the ovaries were washed with PBS, incubated in the dark with secondary antibodies diluted in PBS-T for $3 \mathrm{~h}$ at room temperature and further stained with rhodamine-phalloidin (Biotium, no. 00027) and Hoechst (Sigma) for $20 \mathrm{~min}$. After final washes with PBS, ovaries were mounted with Vectashield. Images were acquired with a Leica SPE confocal microscope and cropped in Image ${ }^{49}$. Depth $(z)$ thresholds were set accordingly to capture the entire architecture. In some cases, brightness and contrast were adjusted in ImageJ (v1.49b). Any changes were applied to all images equally for direct comparison. Antibodies used are provided in Supplementary Table 4.

Protein lysates, fractionation and western blotting. Cells were rinsed with PBS and collected in lysis buffer (50 mM HEPES, $16 \mathrm{mM} \mathrm{NaCl}, 1 \% \mathrm{NP}-40,0.5 \%$ DOC, $0.1 \%$ SDS, $1 \times$ protease inhibitor cocktail (Roche, 04693132001 ) and phosphatase inhibitor cocktail (Sigma, P5726)). Samples were sonicated (15s) for $30 \mathrm{~min}$, spun down at $4^{\circ} \mathrm{C}$ for $20 \mathrm{~min}$ at $12,000 \mathrm{~g}$ and quantified with a DC Protein assay (Biorad, $500-0114)$. Total protein $(50 \mu \mathrm{g})$ was boiled for $10 \mathrm{~min}$ with loading dye (50 mM Tris- $\mathrm{Cl}$ (pH 6.8), $0.3 \%$ SDS, $0.1 \%$ bromophenol blue, $10 \%$ glycerol, $0.4 \%$ ß-mercaptoethanol) and separated by SDS-PAGE followed by electrophoretic transfer to polyvinylidene difluoride (PVDF) membrane (Millipore). Membranes were blocked for $20 \mathrm{~min}$ in PBS-T with 5\% dry milk and primary antibody (in PBS-T with $5 \%$ dry milk) was added for either $1 \mathrm{~h}$ at room temperature or overnight at $4^{\circ} \mathrm{C}$. Membranes were washed three times in PBS-T and incubated with secondary antibody (in PBS-T with 5\% dry milk) for $1 \mathrm{~h}$ at room temperature. After three PBS-T washes, ECL-reagent (Amersham, RPN2132) and X-ray film (Fujifilm, 47410 19236) were used to detect signals. Antibodies used are listed in Supplementary Table 4. Separation of cellular compartments was performed according to the manufacturer's instructions with a Standard Cell Fractionation kit (Abcam, ab109719). Further $\mathrm{HCl}$ treatment of the nuclear fractions was used for chromatin extraction. Alternative chromatin fractionation (Supplementary Fig. 7c) was performed as described previously ${ }^{8}$. U2OS cells were resuspended in buffer A (10 mM HEPES-KOH pH 7.9, $10 \mathrm{mM} \mathrm{KCl,} 1.5 \mathrm{mM} \mathrm{MgCl}_{2}, 340 \mathrm{mM}$ sucrose, $10 \%$ glycerol, $1 \mathrm{mM}$ DTT, $1 \times$ protease inhibitor cocktail (Roche, 04693132001 ) and phosphatase inhibitor cocktail (Sigma, P5726)) and Triton-X100 was added to a final concentration of $0.1 \%$. After incubation on ice for $5 \mathrm{~min}$, samples were centrifuged at $1,300 \mathrm{~g}$ for $4 \mathrm{~min}$. Pelleted nuclei were washed with buffer A, resuspended in buffer B ( $3 \mathrm{mM}$ EDTA, $0.2 \mathrm{mM}$ EGTA, $1 \mathrm{mM}$ DTT, $1 \times$ protease inhibitor cocktail (Roche, 04693132001) and phosphatase inhibitor cocktail (Sigma, P5726)) and lysed for $20 \mathrm{~min}$ on ice. After centrifugation at $1,700 \mathrm{~g}$ for $5 \mathrm{~min}$, the supernatant (nuclear soluble fraction) was collected, and the pellet (chromatin fraction) was washed with buffer B, resuspended in urea buffer $(9 \mathrm{M}$ urea, $50 \mathrm{mM}$ Tris- $\mathrm{HCl} \mathrm{pH} 7.3$ ) and sonicated.

Submitochondrial fractionation. PK treatment of mitochondria was performed as previously described ${ }^{50}$. Briefly, U2OS cells were homogenized in ice-cold MB 
buffer (210 mM mannitol, $70 \mathrm{mM}$ sucrose, $10 \mathrm{mM}$ HEPES-KOH pH 7.4 and $1 \mathrm{mM}$ EDTA) with a glass Dounce homogenizer (40 tight strokes) and mitochondria were obtained by differential centrifugation. To test susceptibility to protease, mitochondria $(100 \mu \mathrm{g})$ were resuspended in MB buffer $(200 \mu \mathrm{l})$, swelling buffer (10 mM HEPES-KOH pH 7.4) or MB +0.2\% (v/v) Triton X-100. PK was added to a final concentration of $50 \mu \mathrm{g} \mathrm{ml}^{-1}$ and the samples were incubated on ice for $30 \mathrm{~min}$. Proteolysis was stopped by adding $2 \mathrm{mM}$ phenylmethylsulfonyl fluoride. Samples were precipitated with trichloroacetic acid (Sigma, T6399) and analysed by SDS-PAGE. Analysis of differential protein retention after FCCP treatment was performed by treating U2OS cells with $25 \mu \mathrm{M}$ FCCP for $24 \mathrm{~h}$. Separation of cellular compartments was performed according to the manufacturer's instructions with a Standard Cell Fractionation kit (Abcam, ab109719). Further $\mathrm{HCl}$ treatment of the nuclear fractions was used for chromatin extraction.

\section{Metabolomic analysis of human cell lines. A combination of liquid} chromatography (LC)-MS and gas chromatography (GC)-MS analysis performed by Metabolon (www.metabolon.com) ${ }^{12}$ was used for the detection of a total of 253 metabolites in cell extracts. Analysis was performed using five independent cultures of control U2OS cells selected with a stable shRNA against GFP (shCont) or EXD2 (shEXD2-22). Cells were collected, snap frozen and metabolites were extracted using a proprietary series of organic and aqueous extractions and divided into two fractions for analysis using LC-MS, LC-MS2 and GC-MS. LC-MS was based on a Waters ACQUITY ultra-performance liquid chromatography system and a Thermo-Finnigan LTQ mass spectrometer, which consisted of an electrospray ionization source and linear ion-trap mass analyser. Samples for GCMS were re-dried under vacuum desiccation for a minimum of $24 \mathrm{~h}$ before analysis and derivatized under dried nitrogen using bistrimethyl-silyl-triflouroacetamide (BSTFA; Sigma, 33024). The GC column was 5\% phenyl and the temperature ramp was from $40^{\circ} \mathrm{C}$ to $300^{\circ} \mathrm{C}$ in a $16 \mathrm{~min}$ period. Samples were analysed on a ThermoFinnigan Trace DSQ fast-scanning single-quadrupole mass spectrometer using electron impact ionization.

For ${ }^{13} \mathrm{C}$ stable isotope tracer analysis, $6 \times 10^{6} \mathrm{U} 2 \mathrm{OS}$ cells stably expressing the indicated shRNA constructs were seeded on $15-\mathrm{cm}$ tissue culture plates in triplicate. The following day, cells were rinsed twice in PBS and $20 \mathrm{ml}$ media (DMEM without glucose, without glutamine, $10 \%$ FBS, $1 \times$ penicillinstreptomycin) containing $5 \mathrm{mM}$ glucose (unlabelled or [ $\left.{ }^{13} \mathrm{C} 6\right]-\mathrm{D}-\mathrm{Glucose}$, Cambridge Isotope Laboratories, CLM-1396-1) or $2 \mathrm{mM}$ glutamine (unlabelled or [U- $\left.{ }^{13} \mathrm{C} 5\right]$-Glutamine, Cambridge Isotope Laboratories, CLM-1822-H-0.1) was added to the cells for $6 \mathrm{~h}$. Cells were collected by trypsinization and $10 \times 10^{6}$ cells were pelleted and snap frozen, after which the metabolites were extracted into solvent by adding $2 \mathrm{ml}$ of ice-cold chloroform:methanol $(2: 1 \mathrm{v} / \mathrm{v})$. The suspension was bath sonicated for $3 \mathrm{~min}$, and $2 \mathrm{ml}$ of cold water was added. Then, $1 \mathrm{ml}$ of chloroform:methanol $(2: 1 \mathrm{v} / \mathrm{v})$ was added to the samples followed by bath sonication for $3 \mathrm{~min}$. Cell lysates were centrifuged $\left(5,000 \mathrm{~g}\right.$ for $15 \mathrm{~min}$ at $\left.4{ }^{\circ} \mathrm{C}\right)$ and the aqueous phase transferred into a new tube. Samples were frozen until analysis using PEPA $^{13}$. PEPA detects the position of carbon labels in isotopically enriched metabolites and quantifies fractional enrichment through the indirect determination of ${ }^{13} \mathrm{C}$-satellite peaks using one-dimensional ${ }^{1} \mathrm{H}$-NMR spectra.

LC-MS analyses. Metabolites were extracted from snap-frozen cell pellets by adding $200 \mu$ l of cold acetonitrile:water $(1: 1)$ with $1 \%$ metaphosphoric acid and $0.1 \%$ formic acid (previously filtered, $1: 1 \mathrm{v} / \mathrm{v}$ ). Samples were vortexed for $30 \mathrm{~s}$ and immersed in liquid $\mathrm{N}_{2}$ to disrupt cell membranes followed by $30 \mathrm{~s}$ of bath sonication; these two steps were repeated three times. Then, samples were incubated at $-20^{\circ} \mathrm{C}$ for $2 \mathrm{~h}$. Finally, samples were centrifuged at $17,000 \mathrm{~g}$ for $15 \mathrm{~min}$ and the supernatant was collected into a LC-MS vial. Samples were injected in a ultra-high performance liquid chromatography system (1290 Agilent) coupled to a triple quadrupole (QqQ) mass spectrometer (6490 Agilent Technologies) operated in multiple reaction monitoring (MRM) and positive (POS) or negative (NEG) electrospray ionization mode. Metabolites were separated using C18-RP (ACQUITY UPLC BEH C18 $1.7 \mu \mathrm{M}$, Waters) chromatography at a flow rate of $0.3 \mathrm{ml} \mathrm{min}^{-1}$. The solvent system was A $\left(20 \mathrm{mM}\right.$ ammonium acetate, $15 \mathrm{mM} \mathrm{NH}_{3}$ in water) and B (acetonitrile:water (95:5)). The linear gradient elution started at $100 \% \mathrm{~A}$ (time $0-2 \mathrm{~min}$ ), $65 \% \mathrm{~A}$ (time $2-5 \mathrm{~min}$ ) and finished at $100 \% \mathrm{~B}$ (time $5.5 \mathrm{~min}$ ). MRM transitions were as follows: reduced glutathione (GSH; NEG, $306 \rightarrow 143.2,128$ ); oxidized glutathione (GSSG; NEG, $611.1 \rightarrow 306.2,143$ ); ATP (POS, $508 \rightarrow 136,410$ ); and ADP (POS $428 \rightarrow 136,348$ ). Cellular ATP content was determined using a luminescence assay (ThermoFisher) according to the manufacturer's instructions, and the relative ATP levels were normalized by the protein content of each sample.

Seahorse Bioanalyzer analysis. Cellular respiration was measured using a Seahorse XF24 Bioanalyzer (Seahorse Biosciences). U2OS and MDA-MB-231 cells were seeded at 50,000 and 75,000 cells per well, respectively, on XF24 V7 PS cell culture microplates (Seahorse Biosciences, 100777-004) and cultured for $16 \mathrm{~h}$ before the analysis. The analysis was performed according to the manufacturer's instructions in DMEM (Seahorse Biosciences, 102353-100) supplemented with 5.5 mM D-glucose and $2 \mathrm{mM} \mathrm{L}$-glutamine for the OCR experiments. For OCR measurements (Mito Stress Test), the following reagents were added: oligomycin
$(1 \mu \mathrm{M})$, FCCP $(0.4 \mu \mathrm{M})$, and rotenone $(1 \mu \mathrm{M})$ and antimycin $\mathrm{A}(1 \mu \mathrm{M})$. For the ECAR analysis (Glycolysis Stress Test), the following reagents were added: glucose $(10 \mathrm{mM})$, oligomycin $(1 \mu \mathrm{m})$ and 2-deoxy-D-glucose $(50 \mathrm{mM})$. For the OCR analysis of individual complexes, digitonin $\left(25 \mu \mathrm{g} \mathrm{ml}^{-1}\right)$ was added to the media to allow cells to permeabilize for approximately $1 \mathrm{~h}$, followed by sequential injections of malate $(2.5 \mathrm{mM})$ and pyruvate $(5 \mathrm{mM})$, rotenone $(1 \mu \mathrm{M})$, succinate $(10 \mathrm{mM})$ and antimycin $(5 \mu \mathrm{M})$. Results were normalized to protein content using a DC Protein assay (Biorad, 500-0114).

\section{BioID analysis of proximity interactions. EXD2 and EXD2-C2 were PCR} amplified from previously described clones, and $d E x d 2$ was amplified from sequence-verified cDNA clones purchased from Gene Synthesis (Life Technologies) with primers containing $5^{\prime}$ AscI or $3^{\prime}$ NotI restriction sites (see Supplementary Table 3 for primers) using KOD Hot Start DNA Polymerase (Millipore) and cycling conditions recommended by the manufacturer: polymerase activation at $95^{\circ} \mathrm{C}$ for $2 \mathrm{~min}$, denaturation at $95^{\circ} \mathrm{C}$ for $20 \mathrm{~s}$, annealing at $55^{\circ} \mathrm{C}$ for $10 \mathrm{~s}$, and extension at $70^{\circ} \mathrm{C}$ for $50 \mathrm{~s}$ (repeated for $40 \mathrm{cycles}$ ). PCR products were purified using a PureLink Quick Gel Extraction kit (Invitrogen) and cloned into pCR2.1-TOPO vector (Invitrogen). Top10 competent Escherichia coli cells (Invitrogen) were transformed with pCR2.1-TOPO clones and colonies were selected in carbenicillin. Constructs were verified by restriction digestion and sequencing (Macrogen) with primers for the TOPO vector (T7 Promoter-F and M13-R). EXD2, EXD2-C2 and $d E x d 2$ were then cut from the pCR2.1-TOPO vector by restriction digestion with AscI (NEB) and NotI-HF (NEB), purified using the PureLink Quick Gel Extraction kit and ligated into pcDNA5/FRT/TO-C-FLAGhBirA* (a gift from B. Raught) using a Quick Ligation Kit (BioLabs). Top10 competent $E$. coli cells (Invitrogen) were transformed and carbenicillin selected, and the constructs were confirmed by restriction digestion and sequencing (Macrogen). For expression in Drosophila S2 cells, dExd2-BirA*-FLAG was PCR amplified and subcloned into the pMK33-C-TAP-FLAG-HA-BD plasmid backbone (Flybase: FBmc0003027).

AD293 cells were seeded and transfected the next day with BirA $^{*}$ vectors using PEI (Polysciences Euro) with or without $50 \mu \mathrm{M}$ biotin (IBIAN Biotechnology, 2-1016-002). For mass spectrometry, Five 15-cm plates per condition were harvested $24 \mathrm{~h}$ post-transfection by trypsinization, washing twice in PBS and snap frozen on dry ice. Cell pellets were lysed in $5 \mathrm{ml}$ modified RIPA buffer $(150 \mathrm{mM}$ Tris- $\mathrm{HCl}$ 7.5, $150 \mathrm{mM} \mathrm{NaCl}, 1 \mathrm{mM}$ EDTA, $1 \mathrm{mM}$ EGTA, 1\% Triton X-100, 0.1\% SDS, 1:500 protease inhibitor cocktail (Sigma-Aldrich), 1:1,000 benzonase (Novagen)) on ice and biotinylated proteins were isolated using streptavidin-sepharose beads (GE Healthcare). Proteins were washed in ammonium bicarbonate and digested with trypsin. Mass spectrometry was performed as previously described ${ }^{51}$.

Sucrose gradient analysis of mitochondrial ribosomes. U2OS cells were treated with $100 \mu \mathrm{g} \mathrm{ml}^{-1} \mathrm{CAP}$ and incubated at $37^{\circ} \mathrm{C}$ for $10 \mathrm{~min}$. Cells were then washed and homogenized in ice-cold STE buffer $(250 \mathrm{mM}$ sucrose, $5 \mathrm{mM}$ Tris- $\mathrm{HCl} \mathrm{pH} 7.5$ and $1 \mathrm{mM}$ EGTA, $2 \mathrm{mM}$ DTT, $100 \mu \mathrm{g} \mathrm{ml}^{-1} \mathrm{CAP}, 20 \mathrm{U} \mathrm{ml}^{-1}$ RNase inhibitors and $1 \times$ complete protease inhibitor) with a glass Dounce homogenizer (40 tight strokes). Mitochondria were obtained by differential centrifugation and loaded $(0.9 \mathrm{mg})$ on a $5 \mathrm{ml}$ linear sucrose gradient $(10-30 \%(\mathrm{v} / \mathrm{v}))$ in a gradient-forming buffer

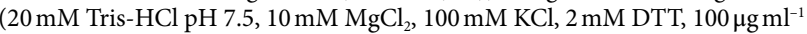
chloramphenicol, $20 \mathrm{U} \mathrm{ml}^{-1} \mathrm{RNase}$ inhibitors and $1 \times$ complete protease inhibitor). The gradients were centrifuged in a Beckman Coulter MLS-50 rotor for $2 \mathrm{~h}$ at $156,500 \mathrm{~g}$ at $4^{\circ} \mathrm{C}$ and fractions of $350 \mu$ l were collected from the top and precipitated with trichloroacetic acid. Samples were then analysed by SDS-PAGE and qRT-PCR.

Analysis of mitochondrial translation. To analyse the rate of mitochondrial translation, experiments were performed essentially as previously described ${ }^{21}$ but with minor modifications. Cells $(400,000)$ were plated on $6-\mathrm{cm}$ plates the day before the experiment. Media was replaced with $2 \mathrm{ml}$ DMEM lacking methionine or cysteine (Life Technologies) with 10\% dialysed FBS (Hyclone) for $20 \mathrm{~min}$. A volume $(100 \mu \mathrm{l})$ of $6 \mathrm{mg} \mathrm{ml}^{-1}$ sterile filtered emetine (Sigma) in PBS was added to the media for $10 \mathrm{~min}$ followed by the addition of $200 \mu \mathrm{Ci} \mathrm{ml}^{-1}$ of ${ }^{35} \mathrm{~S}$ EasyTag (Perkin Elmer) for $1 \mathrm{~h}$. For some experiments, CAP (Sigma) was added at $40 \mu \mathrm{g} \mathrm{ml}^{-}$ to inhibit mitochondrial translation or the antioxidant NAC ( $5 \mathrm{mM}$; Sigma) was added. Labelling media was removed and cells washed twice in PBS followed by a 10 min incubation in DMEM with $10 \%$ FBS and penicillin-streptomycin. Cells were washed three times in PBS and scraped into $1 \mathrm{ml}$ PBS. Following gentle centrifugation at $1,000 \mathrm{~g}$, cell pellets were resuspended in RIPA buffer $(50 \mathrm{mM}$ Tris-HCl pH 7.5, $150 \mathrm{mM} \mathrm{NaCl}, 1 \%$ NP-40, 1 mM MgCl $2,1 \times$ EDTA-free protease inhibitor cocktail (Roche)), nuclei pelleted by centrifugation at $2,000 \mathrm{~g}$ for $10 \mathrm{~min}$ and the protein concentration determined by a modified BCA assay (Biorad). Lysate $(20-40 \mu \mathrm{g})$ was run on a $14 \%$ SDS-PAGE gel and transferred to a PVDF membrane by western blotting. Dried membranes were exposed to a Storage Phosphor Screen (Molecular Dynamics) for 3-8 days and imaged using a Typhoon 8600 Variable Mode Imager (Molecular Dynamics). Contrast was adjusted and band intensity was quantified using Image ${ }^{49}$. In organello Drosophila translation assays were performed as previously described ${ }^{27}$. Briefly, mitochondria were isolated from third-instar larvae and treated with $200 \mu \mathrm{g} \mathrm{ml}^{-1}$ emetine and $100 \mu \mathrm{g} \mathrm{ml}^{-1}$ cyclohexamide to inhibit cytoplasmic translation. Isolated mitochondria $(0.4 \mathrm{mg})$ 
were incubated with $178 \mu \mathrm{Ci}{ }^{35} \mathrm{~S}$ EasyTag (Perkin Elmer) for $1 \mathrm{~h}$ at $30^{\circ} \mathrm{C}$, and equal amounts of mitochondrial protein were loaded on a $14 \%$ SDS-PAGE gel and transferred to a PVDF membrane for western blotting at $100 \mathrm{~V}$ for $1 \mathrm{~h}$. Labelled proteins were visualized by autoradiography as previously described.

Protein purification and enzymatic assays. Recombinant EXD2 (61-621) was used for protein expression because of its improved solubility and yield. Recombinant EXD2 was PCR amplified and cloned into pet28a with an $\mathrm{N}$-terminal His-SUMO tag using BsmBI and XbaI and protein was expressed in E. coli Bl21(DE3)pLysSRARE by the addition of $1 \mathrm{mM}$ isopropyl- $\beta$-D-thiogalactoside (IPTG) at $25^{\circ} \mathrm{C}$ for $3 \mathrm{~h}$. Soluble proteins were purified using nickel affinity resin in $50 \mathrm{mM}$ Tris pH 7.5, $500 \mathrm{mM} \mathrm{NaCl}, 10 \%$ glycerol and eluted with increasing imidazole concentrations (up to $300 \mathrm{mM}$ ). The resulting protein was then further purified by gel filtration using a Superdex 200 10/300 GL column (GE Healthcare) in $50 \mathrm{mM}$ Tris $\mathrm{pH} 7.5,500 \mathrm{mM} \mathrm{NaCl}$, and $10 \%$ glycerol. To confirm the activity was that of EXD2 rather than potential contaminants, mutations D108A and E110A (EXD2-NUC) were generated in the active site by site-directed mutagenesis. In addition, an EXD2-C1/2 mutant was generated (420CxxC to SxxS and $460 \mathrm{CxxC}$ to SxxS) using a QuikChange Lightning Site-Directed Mutagenesis kit (Agilent Technologies, 210518-5). These proteins were then expressed and purified as described for the wild-type protein.

WRN-EXO (1-333) was purified as previously described ${ }^{52}$. Briefly, the protein was expressed in E. coli Bl21(DE3)pLysSRARE by adding $1 \mathrm{mM} \mathrm{IPTG}$ and incubating at $25^{\circ} \mathrm{C}$ for $4 \mathrm{~h}$. Protein was purified on nickel affinity resin in $50 \mathrm{mM}$ Tris pH 7.5, $500 \mathrm{mM} \mathrm{NaCl}$ and $10 \%$ glycerol, and by Q ion exchange chromatography in $50 \mathrm{mM}$ Tris $\mathrm{pH} 7.5$, and $10 \%$ glycerol followed by gel filtration using an S200 column in $50 \mathrm{mM}$ Tris pH 7.5, $500 \mathrm{mM} \mathrm{NaCl}$ and $10 \%$ glycerol.

For enzymatic activity assays, $5-80 \mathrm{nM}$ protein was incubated with $20 \mathrm{nM} 5$ ' FAM (5-carboxyfluorescein) or Cy5 labelled DNA-RNA in $50 \mathrm{mM}$ Tris $\mathrm{pH} 7.5$, $0.5 \mu \mathrm{g} \mathrm{ml}^{-1} \mathrm{BSA}$ and $10 \%$ glycerol with the addition of $10 \mathrm{nM} \mathrm{MnCl}$ or $\mathrm{MgCl}_{2}$. Reactions were incubated at $37^{\circ} \mathrm{C}$ for $30 \mathrm{~min}$ before DNA was denatured by boiling in 35\% formamide. For substrates containing RNA, 50\% formamide was added and no boiling step was used. DNA was separated on an $18 \%$ polyacrylamide urea TBE gel $(21 \mathrm{~W}$ for $2 \mathrm{~h}$ ) and products were visualized using a Fujifilm FLA-5100 image reader. For the blocked $5^{\prime}$ end oligo assay, 0.5 units ExoI (NEB) was used as a positive control and the reaction was carried out in the supplied reaction buffer. Oligonucleotides were annealed to form double-stranded substrates in $10 \mathrm{mM}$ Tris $\mathrm{pH} 7.5$ by boiling followed by slow cooling.

EMSAs. For EMSAs, $20-310 \mathrm{nM}$ protein was incubated with $20 \mathrm{nM} 5^{\prime}$ FAM or Cy5 labelled DNA-RNA in $50 \mathrm{mM}$ Tris $\mathrm{pH} 7.5,0.5 \mu \mathrm{g} \mathrm{ml}^{-1} \mathrm{BSA}$ and $10 \%$ glycerol and incubated on ice for $30 \mathrm{~min}$. Complexes were then separated on an $8 \%$ polyacrylamide gel in $0.5 \times \mathrm{TBE}(75 \mathrm{~V}$ for $80 \mathrm{~min}$ ) and products were visualized using a Fujifilm FLA-5100 image reader. All primers used are listed in Supplementary Table 3

Drosophila stocks and maintenance. D. melanogaster with a transposon insertion mapped to CG6744/dExd2 ( $\mathrm{y}^{1} \mathrm{w}^{67223}$; P\{EPgy2\}CG6744 ${ }^{\mathrm{EY} 03872}$; stock no. 16578) were obtained from the Bloomington Drosophila Stock Center at Indiana University (http://flystocks.bio.indiana.edu/) and the reduced transcript level was confirmed by qRT-PCR as described under 'Quantitative RT-PCR'. Flies were maintained at $25^{\circ} \mathrm{C}$ on a $12 \mathrm{~h}: 12 \mathrm{~h}$ light:dark cycle at constant humidity. Flies were back-crossed to our control yellow white flies for eight generations before performing the analyses reported here.

Drosophila food preparation. For lifespan analysis, different fly-food formulations were prepared as follows: normal food (100 g yeast, $100 \mathrm{~g}$ sugar, $20 \mathrm{~g}$ agar, $30 \mathrm{ml}$ nipagen and $3 \mathrm{ml}$ propionic acid per liter of food); low-sugar food (100 g yeast, $50 \mathrm{~g}$ sugar, $20 \mathrm{~g}$ agar, $30 \mathrm{ml}$ nipagen and $3 \mathrm{ml}$ propionic acid per liter of food); low-yeast food $(50 \mathrm{~g}$ yeast, $100 \mathrm{~g}$ sugar, $20 \mathrm{~g}$ agar, $30 \mathrm{ml}$ nipagen and $3 \mathrm{ml}$ propionic acid per liter of food); antioxidant food ( $100 \mathrm{~g}$ yeast, $100 \mathrm{~g}$ sugar, $20 \mathrm{~g}$ agar, $30 \mathrm{ml}$ nipagen, $3 \mathrm{ml}$ propionic acid and $10 \mathrm{mg} \mathrm{ml}^{-1} \mathrm{NAC}$ per liter of food); and paraquat food (100 g yeast, $100 \mathrm{~g}$ sugar, $20 \mathrm{~g}$ agar, $30 \mathrm{ml}$ nipagen, $3 \mathrm{ml}$ propionic acid and $5 \mathrm{mM}$ paraquat). To prepare each diet, yeast, sucrose and agar were first dissolved in water and boiled for $10 \mathrm{~min}$. After cooling, propionic acid and nipagen were added, and the food was distributed into glass vials and allowed to solidify. Finally, NAC or paraquat was added to the specific diets.

Analysis of Drosophila lifespan, fecundity and development. To assess lifespan, newly hatched flies from each genotype were allowed to mate for $48 \mathrm{~h}$, after which females and males were separated and grouped into 30 flies per vial. Food vials were changed every 3 days, and deaths were scored until all flies were dead. To determine fecundity, age- and diet-specific flies from each genotype were allowed to mate for $4 \mathrm{~h}$ and fecundity was estimated from six independent egg counts. The mean number of eggs per fly was calculated. For the analysis of development, $0-4 \mathrm{~h}$ embryos were collected on grape-juice agar plates. To assess pupariation, 40 newly hatched larvae were transferred to specific-food vials. Once the L3 stage was reached, larvae were monitored and new pupae were counted every $2 \mathrm{~h}$. For size measurements, $0-4 \mathrm{~h}$ embryos and pupae were mounted for imaging and processed using Image $\mathrm{J}^{49}$. Expression data shown in Supplementary Fig. $6 \mathrm{c}$ was obtained from ModENCODE/FlyBase $\mathrm{s}^{5,54}$.

Culture and transfection of Drosophila S2 cells. Drosophila S2 cells were maintained at $25^{\circ} \mathrm{C}$ in Schneider's Drosophila medium (Lonza) supplemented with $10 \%$ (v/v) FBS (Gibco, 10270-500ML) and $50 \mu \mathrm{g} \mathrm{ml}^{-1}$ penicillin-streptomycin (Gibco, 15140-122). S2 cells were transfected using Effectene (Qiagen, 301425) following the manufacturer's protocol.

Oxygen consumption measurement. Oxygen consumption was measured in inverted and permeabilized larval tissues using an Oxygraph-2k (Oroboros) as previously described ${ }^{55}$. Mitochondrial-dependent respiration was measured by supplying the following ETC substrates: $10 \mathrm{mM}$ glutamate, $2 \mathrm{mM}$ malate, $2.5 \mathrm{mM}$ $\mathrm{ADP}$ and $10 \mathrm{mM}$ succinate. Larvae were then stressed with $0.5 \mu \mathrm{M}$ of FCCP to measure maximal respiration, and the ETC was inhibited by adding $0.5 \mu$ $\mathrm{M}$ rotenone and $2.5 \mu \mathrm{M}$ antimycin. Final values were normalized to the protein content of each sample.

TEM. HEK293T cells stably overexpressing EXD2-SF-WT or empty control SF vector (Cont-SF) were grown to $80 \%$ confluency, washed three times in PBS and fixed in $2.5 \%$ glutaraldehyde for $1 \mathrm{~h}$. Cells were detached by scraping and pelleted $\left(4^{\circ} \mathrm{C}, 587 \mathrm{~g}\right.$ in an Eppendorf 5424 rotor for $\left.10 \mathrm{~min}\right)$. Pellets were washed in $0.1 \mathrm{M}$ phosphate buffer (PB) four times for $10 \mathrm{~min}$, stained with $1 \% \mathrm{OsO}_{4}$ for $1 \mathrm{~h}$ and washed again for $10 \mathrm{~min}$ in $0.1 \mathrm{M} \mathrm{PB}$. Cells were dehydrated at $4^{\circ} \mathrm{C}$ using a series of acetone concentrations $(50,70,90,96,100 \%)$ before embedding. Sections were cut with a UCT ultramicrotome (Leica Microsystems) and incubated with blocking buffer ( $5 \%$ and $1 \% \mathrm{BSA}$ ) in $0.01 \mathrm{M}$ PBS solutions for 10 and $1 \mathrm{~min}$, respectively). Grids were incubated for $30 \mathrm{~min}$ at room temperature with polyclonal FLAG antibody (Sigma) diluted 1:10 in blocking buffer (1\% BSA in 0.01 M PBS). After four washes $(0.25 \%$ Tween-20 in $0.01 \mathrm{M}$ PBS for $4 \mathrm{~min}$ and $1 \%$ BSA in $0.01 \mathrm{M}$ PBS for $1 \mathrm{~min}$ ), sections were incubated with immunoglobulin $\mathrm{M}$ anti-rabbit-coupled colloidal gold particles (Jackson, West Grove, USA) diluted in blocking buffer for $15 \mathrm{~min}$, rinsed with water, rinsed in $0.01 \mathrm{M}$ PBS, fixed in $1 \%$ GA in $0.01 \mathrm{M} \mathrm{PBS}$, and rinsed abundantly in double-distilled water. Sections were stained with $1 \%$ potassium permanganate and $1 \%$ uranyl acetate in water for $15 \mathrm{~min}$. Controls for non-specific binding of the colloidal gold-conjugated antibody were performed by omitting the primary antibody. Electron micrographs were obtained using a Jeol JEM 1010 MT electron microscope (Japan) operating at $80 \mathrm{kV}$ in the Electron Microscopy core unit of the Parc Cientific de Barcelona.

Alamar Blue survival assays. Measurements were made according to manufacturer's instructions (Invitrogen, DAL1025). Briefly, cells were seeded at a density of 300 cells per well in 96-well plates and allowed to attach overnight. After completion of drug treatments (7 days), a dilution 1:10 of Alamar Blue reagent in medium was added to each well. After $2-4 \mathrm{~h}$ incubation at $37^{\circ} \mathrm{C}$, fluorescence was measured in a FL600 Microplate Fluorescence Reader (BIOTEK).

Colony formation assays. For colony formation assays, cells were seeded at a density of 300 cells per well in 6-well plates and allowed to attach overnight. After completion of drug treatments (12 days), cells were stained with $0.1 \%$ crystal violet in $20 \%$ methanol. The number of colonies was analysed using Image ${ }^{49}$. Cells were treated with the indicated doses of camptothecin-11 (Sigma, I1406), mitomycin-C (MMC; Sigma, M4287) or olaparib (Selleckchem, AZD2281).

Blue native PAGE. Blue native PAGE analysis was performed according to a previously published protocol $^{56}$. Briefly, $200 \mu \mathrm{g}$ of mitochondria were isolated from U2OS cells, solubilized with $8 \mathrm{~g}$ per gram of digitonin and loaded into a gradient gel. Coomassie staining allowed for the identification of mitochondrial complexes.

MitoString assay. Total RNA was isolated according to the manufacturer's instructions (a PureLink RNA mini kit, Ambion). The MitoString assay was performed as previously described ${ }^{28}$.

2D agarose gel electrophoresis of mitochondrial replication intermediates. $2 \mathrm{D}$ agarose gel electrophoresis was carried out as previously described ${ }^{57}$. Briefly, total DNA was extracted from $4 \times 10^{6}$ cells using phenol:chloroform and PK. Total DNA $(30 \mu \mathrm{g})$ was digested with HinCII (NEB) and DNA was then separated in the first dimension on a $0.4 \%$ TBE agarose gel without ethidium bromide for $18 \mathrm{~h}$ at $1.1 \mathrm{~V} \mathrm{~cm}^{-1}$. The lane was then cut from the gel and rotated $90^{\circ}$ and the DNA was further separated in a $1 \% \mathrm{TBE}$ agarose gel containing $500 \mathrm{ng} \mathrm{m}^{-1}$ ethidium bromide for $6 \mathrm{~h}$ at $260 \mathrm{~mA}, 4^{\circ} \mathrm{C}$. The gel was Southern blotted and DNA was identified using a radioactive probe made using Ready-To-Go dCTP DNA labelling beads (GE Healthcare). The probe was specific to region 16341-151 and was generated by $\mathrm{PCR}^{58}$ (primer sequences are provided in Supplementary Table 3). Labelled membranes were exposed to a phosphor screen that was scanned using a Fujifilm FLA-5100 image reader.

Computational and phylogenetic analysis. Human EXD2 was used as a query in phmmer searches in a database of reference proteomes focusing on the 
UNIPROT reference proteomes having complete genomes. We extracted the EXO domain multiple sequence alignment from the PFAM database (PFAM name is DNA_pol_A_exo1), built a hidden Markov profile from the seed alignment using HMMER (http://hmmer.org/) and searched in our database of Reference proteomes with this profile. Then we collected all the sequences with an EXO domain ( 2300 sequences). The HNH domain was extracted from PFAM (PFAM name $\mathrm{HNH}$ ) and we built a hidden Markov profile for searching our database. EXD2_HUMAN was recovered at a significant e-value above threshold. We next scanned all the sequences for additional domains using hmmscan (from the HMMER suite) to annotate the sequences. Multiple sequence alignments of proteins were conducted using MAFFT in its slow and accurate version (L-IN-SI) ${ }^{59}$ Alignments were visualized in $\mathrm{Belvu}^{60}$ and Jalview ${ }^{61}$. Sequence alignments shown in Supplementary Fig. 1a,c-e were performed using the T-Coffee webserver ${ }^{62}$, the output formatted in Jalview ${ }^{61}$ and modified in Adobe Illustrator. Mitochondrial targeting probability was predicted using MitoProt $\mathrm{II}^{62}$

For the phylogenetic analysis, we conducted two different phylogenies, using only the EXO domain; a large tree of 290 sequences, including a large number of bacterial sequences (available upon request), and a refined tree including representative sequences exhibiting different domain arrangements (Supplementary Fig 1 b). We conducted probabilistic-based phylogenies using MrBayes ${ }^{63}$. A total of four independent chains with four runs were run under a mixed model of evolution over 5 million generations. Twenty-five percent of initial trees were discarded and a total of 1,500,004 trees were sampled. The following specific parameters were used to run the search: prset aamodelpr = mixed; lset rates=gamma; relburnin =yes burningfrac $=0.5$. The tree was annotated using in-house scripts to show the domain distributions associated with the EXO domains in these sequences and visualized using $\mathrm{iTOL}^{64}$. Additional analyses were run using alternative ML-base methods, in particular RA $\times$ ML, giving the same topologies.

For the structural bioinformatic analysis of the EXD2 HNH domain (amino acids 408-470), the EXD2 HNH-like domain was modeled using SCWRL ${ }^{65}$ and superimposed on the PDB structures with the best Fold and Function Assignment (FFAS03) ${ }^{66}$ scores $(5 \times 42$ : Legionella pneumophila DotN: score -15, 5H0M: deepsea thermophilic bacteriophage GVE2 $\mathrm{HNH}$ endonuclease ${ }^{67}$ : score $-12.0,5 \mathrm{MKW}$ : human ZRANB3 HNH domain ${ }^{68}$ : score -11.7 , and 1OGY: heterodimeric nitrate reductase NAPB from Rhodobacter sphaeroides ${ }^{69}$ : score -11.1). Several structures were obtained as reliable templates (between -9 and -16) and the best scoring template in terms of alignment length, \%identity and \%of gaps (5H0M) was used to generate the model presented in Supplementary Fig. 1d.

Statistics and reproducibility. Statistical analysis was performed using Prism 7 and R. An unpaired two-tailed $t$-test was performed when comparing two groups except when stated otherwise. An analysis of variance (ANOVA) test was used when comparing more than two groups, followed by Dunnett's multiple comparison test. For metabolomics data, raw data was median-centered, and metabolite levels compared between shCont and shEXD2-22 groups using a twotailed $t$-test. False discovery rate was controlled using the Benjamini and Hochberg procedure (adjusted $P<0.1$ and a fold change $(\mathrm{FC})>1.3$ in absolute value was used for statistical significance). Data analysis was performed using R version 3.2.0. $\mathrm{MetPa}^{70}$ was used to perform pathway enrichment and topological analysis. Survival or cumulative hazard functions were estimated using the Turnbull method for interval censored data using Kaplan-Meier estimates as a baseline and comparisons performed by proportional-hazard Cox models with interval censored data implemented in R package (https://cran.r-project.org/web/packages/ icenReg/index.html). GSC attrition was analysed with a proportional odds cumulative link model (ordinal regression) implemented in R package (https:// cran.r-project.org/web/packages/ordinal/index.html). $P$ values were adjusted for multiple testing using the Benjimini and Hochberg method and hazard ratios and their corresponding $95 \%$ confidence intervals were computed as a measure of association for data in Figs. 7i, 8a and 8e. Source data and statistical information is shown in Supplementary Table 1. Reproducibility of representative data: Figs. 1a,b and $2 \mathrm{~b}$ : representative of five experiments; Figs. 1d, 3e, 4d, 5e-f, 6b-c, 6e-g, $6 \mathrm{i}, 6 \mathrm{k}-1$, Supplementary Figs. $2 \mathrm{a}, 4 \mathrm{e}, 5 \mathrm{~b}-\mathrm{e}, 5 \mathrm{~g}-\mathrm{i}$, and $5 \mathrm{k}$ : representative of three experiments; Figs. 1e-f, 6d, 6h, 6m, Supplementary Figs. 2f, 5f, 5j, 5m, 6b, 6c, 7e, and 8f: representative of two experiments; Fig. 4a,e: representative of more than 25 cells analysed in two experiments; Fig. 5a: top and middle panels, representative of three experiments, bottom panel representative of two experiments. Figure $5 c$, Supplementary Figs. 4b, 4c, and 4d: representative of seven experiments; Fig. 7h: representative of 30 or more animals per genotype; Fig. $8 \mathrm{~d}$ : representative of 15 or more per animals per genotype; Supplementary Fig. 1f: representative of at least 49 fields from one experiment; Supplementary Fig. 1g: representative of 25 fields from two experiments; Supplementary Figs. $1 \mathrm{~h}$ and 4a: were performed once; Fig. 7e: representative of 30 cells from two experiments.

Life Sciences Reporting Summary. Further information on experimental design is available in the Life Sciences Reporting Summary.
Data availability. Metabolomics data and statistics used for Fig. $2 \mathrm{c}, \mathrm{f}$ are provided in Supplementary Table 1. Raw data for Fig. 2 are available from the Metabolights repository (ID: MTBLS247). An annotated summary of BioID-MS data from Fig. 4 is plotted in Supplementary Table 2, and raw data are available from the MassIVE repository (ID: MSV000081337). Additional source data and statistical information for all plotted figures are provided in Supplementary Table 1 and uncropped blots and gels are provided in Supplementary Figure 9. All other data and materials are available from the corresponding author upon reasonable request.

\section{References}

46. Gloeckner, C. J., Boldt, K., Schumacher, A., Roepman, R. \& Ueffing, M. A novel tandem affinity purification strategy for the efficient isolation and characterisation of native protein complexes. Proteomics 7, 4228-4234 (2007).

47. Naldini, L. et al. In vivo gene delivery and stable transduction of nondividing cells by a lentiviral vector. Science 272, 263-267 (1996).

48. Naviaux, R. K., Costanzi, E., Haas, M. \& Verma, I. M. The pCL vector system: rapid production of helper-free, high-titer, recombinant retroviruses. J. Virol. 70, 5701-5705 (1996)

49. Schindelin, J. et al. Fiji: an open-source platform for biological-image analysis. Nat. Methods 9, 676-682 (2012).

50. Jourdain, A. A. et al. GRSF1 regulates RNA processing in mitochondrial RNA granules. Cell Metab. 17, 399-410 (2013).

51. Gupta, G. D. et al. A dynamic protein interaction landscape of the human centrosome-cilium interface. Cell 163, 1484-1499 (2015).

52. Perry, J. J. et al. WRN exonuclease structure and molecular mechanism imply an editing role in DNA end processing. Nat. Struct. Mol. Biol. 13, 414-422 (2006)

53. Graveley, B. R. et al. The developmental transcriptome of Drosophila melanogaster. Nature 471, 473-479 (2011).

54. Gelbart, W. M. \& Emmert, D. B. FlyBase High Throughput Expression Pattern Data (2013); http://flybase.org/reports/FBrf0221009.html

55. Guitart, T. et al. New aminoacyl-tRNA synthetase-like protein in insecta with an essential mitochondrial function. J. Biol. Chem. 285, 38157-38166 (2010).

56. Wittig, I., Braun, H. P. \& Schagger, H. Blue native PAGE. Nat. Protoc. 1, 418-428 (2006).

57. Reyes, A., Yasukawa, T., Cluett, T. J. \& Holt, I. J. Analysis of mitochondrial DNA by two-dimensional agarose gel electrophoresis. Methods Mol. Biol. 554, 15-35 (2009).

58. Yasukawa, T., Yang, M. Y., Jacobs, H. T. \& Holt, I. J. A bidirectional origin of replication maps to the major noncoding region of human mitochondrial DNA. Mol. Cell 18, 651-662 (2005).

59. Katoh, K. \& Standley, D. M. MAFFT multiple sequence alignment software version 7: improvements in performance and usability. Mol. Biol. Evol. 30, 772-780 (2013)

60. Sonnhammer, E. L. \& Hollich, V. Scoredist: a simple and robust protein sequence distance estimator. BMC Bioinform. 6, 108 (2005).

61. Waterhouse, A. M., Procter, J. B., Martin, D. M., Clamp, M. \& Barton, G. J. Jalview Version 2-a multiple sequence alignment editor and analysis workbench. Bioinformatics 25, 1189-1191 (2009).

62. Claros, M. G. \& Vincens, P. Computational method to predict mitochondrially imported proteins and their targeting sequences. Eur. J. Biochem. 241, 779-786 (1996).

63. Ronquist, F. et al. MrBayes 3.2: efficient Bayesian phylogenetic inference and model choice across a large model space. Syst. Biol. 61, 539-542 (2012).

64. Letunic, I. \& Bork, P. Interactive tree of life (iTOL) v3: an online tool for the display and annotation of phylogenetic and other trees. Nucleic Acids Res. 44, W242-W245 (2016).

65. Krivov, G. G., Shapovalov, M. V. \& Dunbrack, R. L. Jr. Improved prediction of protein side-chain conformations with SCWRL4. Proteins 77, 778-795 (2009).

66. Xu, D., Jaroszewski, L., Li, Z. \& Godzik, A. FFAS-3D: improving fold recognition by including optimized structural features and template re-ranking. Bioinformatics 30, 660-667 (2014).

67. Zhang, L. et al. Structural and functional characterization of deep-sea thermophilic bacteriophage GVE2 HNH endonuclease. Sci. Rep. 7, 42542 (2017).

68. Sebesta, M., Cooper, C. D. O., Ariza, A., Carnie, C. J. \& Ahel, D. Structural insights into the function of ZRANB3 in replication stress response. Nat. Commun. 8, 15847 (2017).

69. Arnoux, P. et al. Structural and redox plasticity in the heterodimeric periplasmic nitrate reductase. Nat. Struct. Biol. 10, 928-934 (2003).

70. Xia, J. \& Wishart, D. S. MetPA: a web-based metabolomics tool for pathway analysis and visualization. Bioinformatics 26, 2342-2344 (2010). 


\section{natureresearch}

Corresponding author(s): Travis H. Stracker

Initial submission

Revised version

\Final submission

\section{Life Sciences Reporting Summary}

Nature Research wishes to improve the reproducibility of the work that we publish. This form is intended for publication with all accepted life science papers and provides structure for consistency and transparency in reporting. Every life science submission will use this form; some list items might not apply to an individual manuscript, but all fields must be completed for clarity.

For further information on the points included in this form, see Reporting Life Sciences Research. For further information on Nature Research policies, including our data availability policy, see Authors \& Referees and the Editorial Policy Checklist.

\section{- Experimental design}

\section{Sample size}

Describe how sample size was determined.

Evaluation of the data distribution and variation was performed in order to make sure the chosen statistical tests were appropriate. No statistical method was used to predetermine sample size.

\section{Data exclusions}

Describe any data exclusions.

3. Replication

Describe whether the experimental findings were reliably reproduced.

\section{Randomization}

Describe how samples/organisms/participants were allocated into experimental groups.

\section{Blinding}

Describe whether the investigators were blinded to group allocation during data collection and/or analysis.
No samples or animals were excluded from the analyses.

All experiments were performed at least 3 times with similar results with the exception of Figs. 1e-f, 6d, 6h, 6m, Supplementary Figs. 2f, 5f, 5j, 5m, 6c, $6 \mathrm{~d}$ that were performed 2 times with similar results and Supplementary Fig. 1h, 4a that were performed a single time. Details are provided in the Statistics and Reproducibility section of the Materials and Methods.

The experiments were not randomized.

Generally, the investigators were not blinded to allocation during experiments and outcome assessment. MS experiments were run blinded to the operators.

Note: all studies involving animals and/or human research participants must disclose whether blinding and randomization were used.

6. Statistical parameters

For all figures and tables that use statistical methods, confirm that the following items are present in relevant figure legends (or in the Methods section if additional space is needed).

n/a Confirmed

The exact sample size $(n)$ for each experimental group/condition, given as a discrete number and unit of measurement (animals, litters, cultures, etc.)

A description of how samples were collected, noting whether measurements were taken from distinct samples or whether the same sample was measured repeatedly

A statement indicating how many times each experiment was replicated

The statistical test(s) used and whether they are one- or two-sided (note: only common tests should be described solely by name; more complex techniques should be described in the Methods section)

$\bigotimes$ A description of any assumptions or corrections, such as an adjustment for multiple comparisons

$\bigotimes$ The test results (e.g. $P$ values) given as exact values whenever possible and with confidence intervals noted

A clear description of statistics including central tendency (e.g. median, mean) and variation (e.g. standard deviation, interquartile range) Clearly defined error bars 
Policy information about availability of computer code

\section{Software}

Describe the software used to analyze the data in this study.

No commercial or custom code was used. Graphpad Prism 7 was used to plot data and for some statistical analysis (ANOVA and t-test). Other statistical analysis was performed by the IRB Barcelona Biostatistics/Bioinformatics core facility using R as described in the Materials and Methods.

For manuscripts utilizing custom algorithms or software that are central to the paper but not yet described in the published literature, software must be made available to editors and reviewers upon request. We strongly encourage code deposition in a community repository (e.g. GitHub). Nature Methods guidance for providing algorithms and software for publication provides further information on this topic.

\section{- Materials and reagents}

Policy information about availability of materials

8. Materials availability

Indicate whether there are restrictions on availability of unique materials or if these materials are only available for distribution by a for-profit company.

\section{Antibodies}

Describe the antibodies used and how they were validated for use in the system under study (i.e. assay and species).

\section{Eukaryotic cell lines}

a. State the source of each eukaryotic cell line used.

b. Describe the method of cell line authentication used.

c. Report whether the cell lines were tested for mycoplasma contamination.

d. If any of the cell lines used are listed in the database of commonly misidentified cell lines maintained by ICLAC, provide a scientific rationale for their use.
All materials are available to the scientific community upon reasonable request.

All antibodies are listed in Supplementary Table 4 with species, company/catalog\# and dilution used per application. The EXD2 antibody was validated on human CRSIPR KO cells or cells expressing specific shRNAs against EXD2. Other antibodies were validated by extensive use in previous publications, control experiments (behavior in fractionation, localization) or by the distributor (TOM20, H3, ATP5A, NDUFA9, VDAC, Cytochrome-C, GFP, OXPHOS cocktail, GAPDH, Lamin, Hts, RPA2, ND6, MRPL3, MRPL12, DAP3).

AD293 cells were purchased from Stratagene. MDA-MB-231 cells were obtained from the lab of Dr. Roger Gomis and U2OS and RPE1 cell lines were purchased from ATCC.

Morphology, behavior and several gene expression parameters were consistent with their identity but genetic validation was not carried out.

Cells were routinely tested for mycoplasma by PCR (primers provided in Table 3) and found negative.

No. commonly misidentified cell lines were used

\section{- Animals and human research participants}

Policy information about studies involving animals; when reporting animal research, follow the ARRIVE guidelines

\section{Description of research animals}

Provide details on animals and/or animal-derived No vertebrate animals were used.

materials used in the study.

\section{Policy information about studies involving human research participants}

\section{Description of human research participants}

Describe the covariate-relevant population characteristics of the human research participants.

The study did not involve human participants. 


\section{natureresearch}

\section{Flow Cytometry Reporting Summary}

Form fields will expand as needed. Please do not leave fields blank.

\section{- Data presentation}

For all flow cytometry data, confirm that:

$\bigotimes 1$. The axis labels state the marker and fluorochrome used (e.g. CD4-FITC).

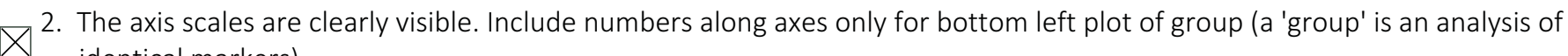
identical markers).

$\bigotimes$ 3. All plots are contour plots with outliers or pseudocolor plots.

$\bigotimes 4$. A numerical value for number of cells or percentage (with statistics) is provided.

- Methodological details

5. Describe the sample preparation.

6. Identify the instrument used for data collection.

7. Describe the software used to collect and analyze the flow cytometry data.

8. Describe the abundance of the relevant cell populations within post-sort fractions.

9. Describe the gating strategy used.
150,000 U2OS or MDA-MB-231 cells were stained per sample with MitoTracker Green FM, MitoTracker Red or MitoSox Red. Cells were trypsinized and washed with warm PBS once, stained with DAPI (to identify dead cells) and analyzed by flow cytometry immediately. No fixation was used.

\section{Gallios, Beckman Coulter.}

Data was collected using Kaluza for Gallios acquisition software (Beckman Coulter) and anlayzed using FlowJo v9.9.6 and Excel. Data was plotted and statistically analyzed in Prism7.

No sorting was performed.

Dead cells were gated with DAPI staining and dye stained populations were validated by comparing to control treated samples: FCCP (MitoTracker) or Paraquat (MitoSox). Examples are shown in Supplementary Figure 8.

Tick this box to confirm that a figure exemplifying the gating strategy is provided in the Supplementary Information. $\bigotimes$ 


\section{natureresearch}

\section{Flow Cytometry Reporting Summary}

Form fields will expand as needed. Please do not leave fields blank.

\section{- Data presentation}

For all flow cytometry data, confirm that:

$\bigotimes 1$. The axis labels state the marker and fluorochrome used (e.g. CD4-FITC).

$X^{2}$. The axis scales are clearly visible. Include numbers along axes only for bottom left plot of group (a 'group' is an analysis of identical markers).

$\bigotimes$ 3. All plots are contour plots with outliers or pseudocolor plots.

$\bigotimes 4$. A numerical value for number of cells or percentage (with statistics) is provided.

\section{- Methodological details}

5. Describe the sample preparation.

150,000 U2OS or MDA-MB-231 cells were stained per sample with MitoTracker Green FM, MitoTracker Red or MitoSox Red. Cells were trypsinized and washed with warm PBS once, stained with DAPI (to identify dead cells) and analyzed by flow cytometry immediately. No fixation was used.

6. Identify the instrument used for data collection.

7. Describe the software used to collect and analyze the flow cytometry data.

8. Describe the abundance of the relevant cell populations within post-sort fractions.

9. Describe the gating strategy used.
Gallios, Beckman Coulter.

Data was collected using Kaluza for Gallios acquisition software (Beckman Coulter) and anlayzed using FlowJo v9.9.6 and Excel. Data was plotted and statistically analyzed in Prism7.

No sorting was performed.

Dead cells were gated with DAPI staining and dye stained populations were validated by comparing to control treated samples: FCCP (MitoTracker) or Paraquat (MitoSox). Examples are shown in Supplementary Figure 8.

Tick this box to confirm that a figure exemplifying the gating strategy is provided in the Supplementary Information. $\bigotimes$ 